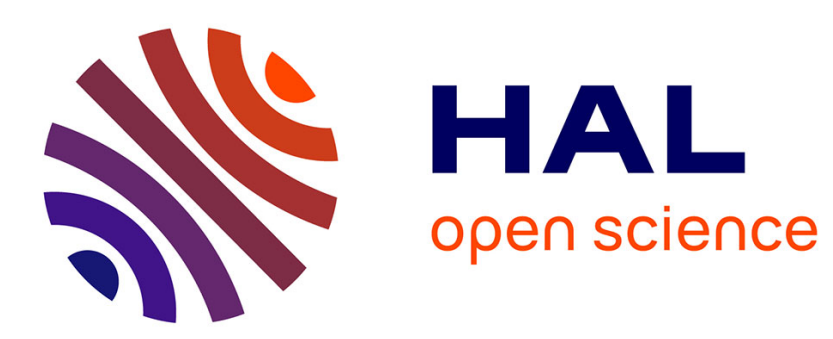

\title{
Pressure correction staggered schemes for barotropic one-phase and two-phase flows
}

\author{
Walid Kheriji, Raphaele Herbin, Jean-Claude Latché
}

\section{To cite this version:}

Walid Kheriji, Raphaele Herbin, Jean-Claude Latché. Pressure correction staggered schemes for barotropic one-phase and two-phase flows. Computers and Fluids, 2013, 88, pp.524-542. 10.1016/j.compfluid.2013.09.022 . hal-00875348

\section{HAL Id: hal-00875348 https://hal.science/hal-00875348}

Submitted on 21 Oct 2013

HAL is a multi-disciplinary open access archive for the deposit and dissemination of scientific research documents, whether they are published or not. The documents may come from teaching and research institutions in France or abroad, or from public or private research centers.
L'archive ouverte pluridisciplinaire HAL, est destinée au dépôt et à la diffusion de documents scientifiques de niveau recherche, publiés ou non, émanant des établissements d'enseignement et de recherche français ou étrangers, des laboratoires publics ou privés. 


\title{
Pressure correction staggered schemes for barotropic one-phase and two-phase flows
}

\author{
W. Kheriji*†, R. Herbin* and J.-C. Latché ${ }^{\dagger}$ \\ * Aix-Marseille Université, CNRS, Centrale Marseille, LATP, UMR 7353, 13453 Marseille, France. \\ † Institut de Radioprotection et de Sûreté Nucléaire (IRSN), France \\ herbin@cmi.univ-mrs.fr, [walid.kheriji, jean-claude.latche]@irsn.fr
}

\section{Summary}

We assess in this paper the capability of a pressure correction scheme to compute shock solutions of the homogeneous model for barotropic two-phase flows. This scheme is designed to inherit the stability properties of the continuous problem: the unknowns (in particular the density and the dispersed phase mass fraction $y$ ) are kept within their physical bounds, and the entropy of the system is conserved, thus providing an unconditional stability property. In addition, the scheme keeps the velocity and pressure constant through contact discontinuities. These properties are obtained by coupling the mass balance and the transport equation for $y$ in an original pressure correction step. The space discretization is staggered; the numerical schemes which are considered are the Marker-And Cell (MAC) finite volume scheme and the nonconforming low-order Rannacher-Turek and Crouzeix-Raviart finite element approximation. In either case, a finite volume technique is used for all convection terms. Numerical experiments performed here show that, provided that a sufficient dissipation is introduced in the scheme, it converges to the (weak) solution of the continuous hyperbolic system. Observed orders of convergence for 1D Riemann problems as a function of the mesh and time step at constant CFL number vary with the studied case, and the CFL number and on the regularity of the solution. They range from 0.5 to greater than 1 for the velocity and the pressure; in most cases, the density and mass fraction converge with a 0.5 order. Finally, the scheme shows a satisfactory behaviour up to large CFL numbers.

Keywords : Barotropic Euler Equations, Shallow Water Equations, Staggered Discretizations, Finite Volumes, Finite Elements, Pressure Correction Schemes. 
Pressure correction staggered schemes for barotropic one-phase and two-phase flows

\section{Contents}

1 Introduction $\quad 2$

2 The pressure correction scheme 5

2.1 Time semi-discretization . . . . . . . . . . . . . . . . . . . . . 5

2.2 Discrete spaces and unknowns . . . . . . . . . . . . . . . . . . . . . . . 6

2.3 Discrete equations ............................ 7

3 Riemann problems $\quad 11$

3.1 Sod shock tube problem . . . . . . . . . . . . . . . . . . . . . . 12

3.2 Two-fluid shock tube . . . . . . . . . . . . . . . . . . . . . . 18

3.2.1 First case: shock - contact discontinuity - shock . . . . . . . . . . . . . . 18

3.2.2 Second case: rarefaction-contact discontinuity-rarefaction . . . . . . . . . 24

$\begin{array}{lll}4 & \text { Wind tunnel with a step } & 27\end{array}$

5 Flow past a cylinder $\quad 31$

5.1 A low Mach number case . . . . . . . . . . . . . . . . . . . . 31

5.2 A compressible case . . . . . . . . . . . . . . . . . . . . . . . 32

6 Conclusion $\quad 36$

\section{Introduction}

We address in this paper the following homogeneous two-phase flow model, describing for instance the flow of a liquid-gas mixture:

$$
\begin{aligned}
& \partial_{t} \rho+\operatorname{div}(\rho \boldsymbol{u})=0, \\
& \partial_{t} z+\operatorname{div}(z \boldsymbol{u})=0, \\
& \partial_{t}(\rho \boldsymbol{u})+\operatorname{div}(\rho \boldsymbol{u} \otimes \boldsymbol{u})+\nabla p-\operatorname{div}(\boldsymbol{\tau}(\boldsymbol{u}))=0,
\end{aligned}
$$

where $\partial_{t}$ is the time derivative, $\rho, \boldsymbol{u}$ and $p$ are the (average) density, velocity and pressure in the flow, $z$ stands for the partial density of one of the two phases, let us say the gas phase. The first two equations, (1) and (2), are the mixture and the gas mass balance equations respectively, and the third equation (3) is the mixture momentum balance equation. The tensor $\boldsymbol{\tau}$ is the viscous part of the stress tensor, given by the following expression:

$$
\boldsymbol{\tau}(\boldsymbol{u})=\mu\left(\boldsymbol{\nabla} \boldsymbol{u}+\nabla^{t} \boldsymbol{u}\right)-\frac{2}{3} \mu(\operatorname{div} \boldsymbol{u}) \boldsymbol{I},
$$

where the viscosity $\mu$ stands for a non-negative real number and $\boldsymbol{I}$ for the identity tensor of $\mathbb{R}^{d \times d}$. The problem is defined over an open bounded connected subset $\Omega$ of $\mathbb{R}^{d}, d \leq 3$, and over a finite time interval $(0, T)$. We suppose that suitable initial and boundary conditions are provided for $\rho, \boldsymbol{u}$ and $z$; in particular, $\rho, \boldsymbol{u}$ and $z$ are supposed to be prescribed at the inflow boundaries, and the prescribed values are taken such that the phasic densities remain positive.

In order to close the problem, we need an additional relation, which results from the combination of the mixture equation of state and the phasic equations of state, i.e. relations satisfied by the 
Pressure correction staggered schemes for barotropic one-phase and two-phase flows

density of each phase. Let us begin with the latter ones. The liquid density $\rho_{\ell}$ is supposed to be constant, and the gas density $\varrho_{g}$ is assumed to depend on the pressure only:

$$
\rho_{g}=\varrho_{g}(p)
$$

where $\varrho_{g}$ is defined and increasing over $[0,+\infty), \varrho_{g}(0)=0$ and $\lim _{s \rightarrow+\infty} \varrho_{g}(s)=+\infty$. Such a flow (i.e. a flow where phasic densities are functions of the pressure only) is usually referred to as a barotropic flow. Finally, the mixture equation of state is usually written:

$$
\rho=(1-\alpha) \rho_{\ell}+\alpha \rho_{g},
$$

where $\alpha$ is called the void fraction (the volume of gas per specific volume) and is related to the unknowns of the problem by:

$$
z=\alpha \rho_{g},
$$

which yields the needed additional relation linking the unknowns of the problem together:

$$
\rho=\left[1-\frac{z}{\varrho_{g}(p)}\right] \rho_{\ell}+z
$$

If we introduce the gas mass fraction $y=z / \rho$ (the gas mass per specific mass), we get equivalently:

$$
\rho=\frac{1}{\frac{y}{\rho_{g}}+\frac{1-y}{\rho_{\ell}}} .
$$

We now recall some estimates which are satisfied, at least formally, by the solution of System (1)-(3). Equation (1) shows that $\rho$ remains non-negative at all time. Replacing $z$ by $\rho y$ in the gas mass balance equation (2) and using the mass balance equation (1), we get:

$$
\partial_{t}(\rho y)+\nabla \cdot(\rho y \boldsymbol{u})=\rho\left(\partial_{t} y+\boldsymbol{u} \cdot \nabla y\right)=0 .
$$

Let us suppose that $\rho$ does not vanish (which is not necessarily true at the continuous level, since $\operatorname{div} \boldsymbol{u}$ is not bounded in $\mathrm{L}^{\infty}(\Omega)$, but will be true at the discrete level). Then this relation implies that $y$ satisfies the transport equation $\partial_{t} y+\boldsymbol{u} \cdot \nabla y=0$, and thereby obeys a maximum principle. Specifically, if the initial and boundary conditions for $\rho$ and $z$ are such that $y \in[\varepsilon, 1]$ at $t=0$, where $0<\varepsilon \leq 1$ (which excludes purely liquid zones at the initial time), we obtain that $y$ remains in the interval $[\varepsilon, 1]$ at all times. From (8) and the fact that $\rho>0$, we can deduce that $\rho \in\left[\min \left(\rho_{\ell}, \rho_{g}\right), \max \left(\rho_{\ell}, \rho_{g}\right)\right]$ and, now from $(6), \alpha \in(0,1]$, so $\rho_{g}=z / \alpha>0$ and, since $\varrho_{g}$ is one-to-one from $(0,+\infty)$ to itself, there exists a positive pressure $p$ such that $\rho_{g}=\varrho_{g}(p)$.

Let us now define the function $\mathcal{P}$, from $(0,+\infty)$ to $\mathbb{R}$, as a primitive of $s \mapsto \wp_{g}(s) / s^{2}$, where $\wp_{g}=\varrho_{g}^{-1}$. Then, if we suppose that the velocity is prescribed to zero at the boundary, the solution to System (1)-(3) satisfies:

$$
\frac{d}{d t} \int_{\Omega}\left[\frac{1}{2} \rho|\boldsymbol{u}|^{2}+z \mathcal{P}\left(\varrho_{g}(p)\right)\right] \mathrm{d} \boldsymbol{x} \leq 0 .
$$

The quantity $z \mathcal{P}\left(\varrho_{g}(p)\right)$ is often called the Helmholtz energy, $\frac{1}{2} \rho|\boldsymbol{u}|^{2}$ the kinetic energy and their sum is the entropy of the system. Since the function $\mathcal{P}$ is increasing, Inequality (9) provides an estimate on the solution.

When $\mu=0$, the viscous term $\tau(u)$ vanishes and the system (1)-(3) becomes hyperbolic, with a simple wave structure (see [14] for a comprehensive presentation). The solution to the (1D) 
Pressure correction staggered schemes for barotropic one-phase and two-phase flows

Riemann problems [23] always involves a contact discontinuity, moving at a celerity equal to the velocity $u$, and two genuinely non-linear (shock or rarefaction) waves, moving at the celerity $u-c$ and $u+c$, where $c$ stands for the sound velocity in the mixture (see Relation (19) in Section 4 below). Through the contact discontinuity, the pressure and velocity are kept constant, and $z$, $\rho$ or $y$ may be discontinuous. The existence of this wave may be inferred by just checking that, provided that this is consistent with initial and boundary conditions, a solution to the system with constant velocity and pressure exists: indeed, from the first form of (7), it may be seen that $\rho$ and $z$ are linked by an affine relation with constant coefficients (for a constant pressure); (1) and (2) then boil down to the same transport equation (with a constant velocity) and (3) is trivially satisfied.

Finally, note that, since $y$ satisfies a transport equation, if $y=1$ at the initial time (everywhere in the domain) and at inflow boundaries (at all time), the solution satisfies $y=1$ for all $\boldsymbol{x} \in \Omega$ and $t \in(0, T)$. In such a case, System (1)-(3) boils down to the governing equations of barotropic one-phase flows; for the particular equation of state $\varrho_{g}(s)=s^{1 / 2}$, we recover in one or two dimensions the usual shallow-water equations.

The use of pressure correction schemes for compressible single phase flows seems to be widespread (see e.g. [15] for the seminal work and [28] for a comprehensive introduction). Indeed, pressure correction schemes are partly implicit, thereby ensuring some stability with respect to the time step together with introducing a decoupling of the equations which helps the numerical solution of the nonlinear systems. Extensions to multi-phase flows are scarcer and seem to be restricted to iterative algorithms, often similar in spirit to the usual SIMPLE algorithm for incompressible flows $[27,22,20]$. In this paper, we perform a numerical study of a non-iterative pressurecorrection scheme introduced in [10], based on a low order finite element and a finite volume discretization, which enjoys the following properties:

(i) the scheme has at least one solution, and any solution satisfies the above listed "discretemaximum-based" estimates: $\rho>0$, the gas mass fraction $y$ satisfies a discrete maximum principle (so $0 \leq y \leq 1)$, and $p>0$.

(ii) the scheme is unconditionally stable, in the sense that its solution(s) satisfies a discrete analogue of Inequality (9),

(iii) the pressure and velocity are kept constant through contact discontinuities.

In addition, the scheme is conservative for $\rho$ and $z$. As in the continuous case, thanks to the fact that $y$ is kept constant if it is consistent with the initial and boundary conditions, it also copes with one-phase barotropic flows, a particular case of which may be formally identified to the shallow water equations. Finally, the scheme boils down to the usual projection scheme for incompressible flows (obtained in the present framework when $y=0$ or, asymptotically, when the function $\varrho_{g}$ becomes constant), and is indeed routinely used for the computation of low Mach number flows, as, for instance, classical bubble columns of chemical engineering processes or two-phase flows encountered in nuclear safety studies. Its accuracy was assessed for smooth solutions in [10], and the aim of the present paper is to check its convergence and accuracy in non-diffusive cases, for weak solutions with discontinuities.

The paper is organized as follows. We first present the scheme (Section 2). Then we study several Riemann problems, first one-phase $(y=1)$ (Section 3.1) then two-phase: in this latter case, we first address a flow that involves only a contact discontinuity and shocks (Section 3.2.1), and finally a flow with rarefaction waves (Section 3.2.2). Finally, we assess in Sections 4 and 
Pressure correction staggered schemes for barotropic one-phase and two-phase flows

5 the behaviour of the scheme on two-dimensional test cases; results of Section 5 also show that the scheme is able to capture the solution of an almost incompressible (or very low Mach number) problem.

\section{The pressure correction scheme}

\subsection{Time semi-discretization}

Let us consider a partition $0=t_{0}<t_{1}<\ldots<t_{N}=T$ of the time interval $(0, T)$, which is supposed uniform. Let $\delta t=t_{n+1}-t_{n}$ for $n=0,1, \ldots, N-1$ be the constant time step. In a time semi-discrete setting, denoting by $\rho^{-1}$ and $u^{0}$ initial guesses for the density and velocity, the algorithm proposed in this paper is the following.

0 - Initialization - Compute $\rho^{0}$ by solving the following mass balance equation:

$$
\frac{1}{\delta t}\left(\rho^{0}-\rho^{-1}\right)+\operatorname{div}\left(\rho^{0} \boldsymbol{u}^{0}\right)=0 .
$$

Then, for $n \geq 0$ :

1 - Prediction step - Solve the following linearized momentum balance equation for $\tilde{\boldsymbol{u}}^{n+1}$ :

$$
\frac{1}{\delta t}\left(\rho^{n} \tilde{\boldsymbol{u}}^{n+1}-\rho^{n-1} \boldsymbol{u}^{n}\right)+\operatorname{div}\left(\rho^{n} \tilde{\boldsymbol{u}}^{n+1} \otimes \boldsymbol{u}^{n}\right)+\nabla p^{n}-\operatorname{div}\left(\boldsymbol{\tau}\left(\tilde{\boldsymbol{u}}^{n+1}\right)\right)=0 .
$$

2 - Pressure correction step - Solve (simultaneously) the following non-linear equations for $p^{n+1}, \boldsymbol{u}^{n+1}, \rho^{n+1}$ and $z^{n+1}$ :

$$
\begin{aligned}
& \frac{\rho^{n}}{\delta t}\left(\boldsymbol{u}^{n+1}-\tilde{\boldsymbol{u}}^{n+1}\right)+\nabla\left(p^{n+1}-p^{n}\right)=0, \\
& \frac{1}{\delta t}\left(\rho^{n+1}-\rho^{n}\right)+\operatorname{div}\left(\rho^{n+1} \boldsymbol{u}^{n+1}\right)=0, \\
& \frac{1}{\delta t}\left(z^{n+1}-z^{n}\right)+\operatorname{div}\left(z^{n+1} \boldsymbol{u}^{n+1}\right)=0, \\
& \rho^{n+1}=\varrho\left(p^{n+1}, z^{n+1}\right)=z^{n+1}\left[1-\frac{\rho_{\ell}}{\varrho_{g}\left(p^{n+1}\right)}\right]+\rho_{\ell} .
\end{aligned}
$$

Step 1 is the usual prediction step for the velocity, which consists in solving the momentum balance equation (3) with the beginning-of-step pressure. Step 2 is the pressure correction step. Its resolution is performed by combining equations (12a) and (12b), therefore obtaining a non-linear elliptic problem for the pressure, which reads in the time semi-discrete setting:

$$
\begin{aligned}
\frac{1}{\delta t^{2}}\left(\rho^{n+1}-\rho^{n}\right)-\operatorname{div}\left[\frac{\rho^{n+1}}{\rho^{n}} \nabla\left(p^{n+1}-p^{n}\right)\right] & =-\frac{1}{\delta t} \operatorname{div}\left(\rho^{n+1} \tilde{\boldsymbol{u}}^{n+1}\right), \\
& \text { with } \rho^{n+1}=\varrho\left(p^{n+1}, z^{n+1}\right)=z^{n+1}\left[1-\frac{\rho_{\ell}}{\varrho_{g}\left(p^{n+1}\right)}\right]+\rho_{\ell} .
\end{aligned}
$$

Note that, for a given space discretization, this equation must be established at the algebraic level [10], with the discrete equivalent manipulations which were necessary to derive it at the 
Pressure correction staggered schemes for barotropic one-phase and two-phase flows

continuous level (i.e. multiplying the first equation by $\rho^{n+1} / \rho^{n}$, taking its divergence and subtracting to the second relation).

Two features are unusual in this algorithm. The first one is the time-shift of the densities in the prediction step: thanks to this time-shift, the densities satisfy $(12 \mathrm{~b})$ of the preceding correction step and therefore the convection operator vanishes for constant velocities (i.e. $\tilde{u}^{n+1}=\mathrm{cste}$ ), which is shown to be a necessary condition to ensure the conservation of the kinetic energy $[8,1]$. Second, the pressure correction step couples the mixture and dispersed phase mass balance equations (12b) and (12c); this coupling preserves the affine relation between $\rho^{n+1}$ and $z^{n+1}$ through the equation of state (12d), with coefficients only depending on the pressure (taken at the same time level). Thus, as in the continuous case, both equations boil down to only one relation when the pressure and the velocity are constant (precisely speaking, when the pressure is constant and the velocity is divergence-free); consequently, the arguments necessary to obtain solutions with constant velocity and pressure (i.e. contact discontinuity waves) still hold at the discrete level.

\section{$2.2 \quad$ Discrete spaces and unknowns}

Let $\mathcal{M}$ be a decomposition of the domain $\Omega$, supposed to be regular in the usual sense of the finite element literature (e.g. [5]). The cells may be:

- for a general domain $\Omega$, either non-degenerate quadrilaterals $(d=2)$ or hexahedra $(d=3)$ or simplices, both type of cells being possibly combined in a same mesh,

- for a domain the boundaries of which are hyperplanes normal to a coordinate axis, rectangles $(d=2)$ or rectangular parallelepipeds $(d=3)$ (the faces of which, of course, are then also necessarily normal to a coordinate axis).

By $\mathcal{E}$ and $\mathcal{E}(K)$ we denote the set of all faces $\sigma$ of the mesh and of the element $K \in \mathcal{M}$ respectively. The set of faces included in the boundary of $\Omega$ is denoted by $\mathcal{E}_{\text {ext }}$ and the set of internal faces (i.e. $\mathcal{E} \backslash \mathcal{E}_{\text {ext }}$ ) is denoted by $\mathcal{E}_{\text {int }}$. For each internal face of the mesh $\sigma=K \mid L, \boldsymbol{n}_{K L}$ stands for the normal vector to $\sigma$, oriented from $K$ to $L$. The outward normal vector to a face $\sigma$ of $K$ is denoted by $\boldsymbol{n}_{K, \sigma}$. For $K \in \mathcal{M}$ and $\sigma \in \mathcal{E}$, we denote by $|K|$ the measure of $K$ and by $|\sigma|$ the $(d-1)$-dimensional measure of the face $\sigma$. For $1 \leq i \leq d$, we denote by $\mathcal{E}^{(i)} \subset \mathcal{E}$ the subset of the faces of $\mathcal{E}$ which are perpendicular to the $i^{\text {th }}$ unit vector of the canonical basis of $\mathbb{R}^{d}$.

The space discretization is staggered, using either the Marker-And Cell (MAC) scheme [16, 15], or nonconforming low-order finite element approximations, namely the Rannacher-Turek element (RT) [25] for quadrilateral or hexahedric meshes, or the lowest degree Crouzeix-Raviart (CR) element $[6]$ for simplicial meshes.

For all these space discretizations, the degrees of freedom for the pressure, the density and the variables $y$ and $z$ are associated to the cells of the mesh $\mathcal{M}$ : the degrees of freedom are therefore

$$
\left\{p_{K}, \rho_{K}, y_{K}, z_{K}, K \in \mathcal{M}\right\}
$$

Let us then turn to the degrees of freedom for the velocity.

- Rannacher-Turek or Crouzeix-Raviart discretizations - The approximation for the velocity is nonconforming (the discrete functions are discontinuous through a face, but the jump of their integral is imposed to be zero); the degrees of freedom for the velocities are located at the center of the faces of the mesh, and we choose the version of the element 
Pressure correction staggered schemes for barotropic one-phase and two-phase flows

where they represent the average of the velocity through a face. The set of degrees of freedom reads:

$$
\left\{u_{\sigma, i}, \sigma \in \mathcal{E}, 1 \leq i \leq d\right\}
$$

We denote by $\varphi_{\sigma}^{(i)}$ the vector shape function associated to $u_{\sigma, i}$, which, by definition, reads $\boldsymbol{\varphi}_{\sigma}^{(i)}=\varphi_{\sigma} \boldsymbol{e}^{(i)}$, where $\varphi_{\sigma}$ is the Rannacher-Turek (RT) or Crouzeix-Raviart (CR) scalar shape function and $\boldsymbol{e}^{(i)}$ is the $i^{\text {th }}$ vector of the canonical basis of $\mathbb{R}^{d}$, and we define $u_{\sigma}$ by $\boldsymbol{u}_{\sigma}=\sum_{i=1}^{d} u_{\sigma, i} \boldsymbol{e}^{(i)}$. With these definitions, we have the identity:

$$
\boldsymbol{u}(\boldsymbol{x})=\sum_{\sigma \in \mathcal{E}} \sum_{i=1}^{d} u_{\sigma, i} \boldsymbol{\varphi}_{\sigma}^{(i)}(\boldsymbol{x})=\sum_{\sigma \in \mathcal{E}} \boldsymbol{u}_{\sigma} \varphi_{\sigma}(\boldsymbol{x}), \quad \text { for a.e. } \boldsymbol{x} \in \Omega .
$$

- MAC discretization. The degrees of freedom for the $i^{\text {th }}$ component of the velocity, defined at the centres of the face $\sigma \in \mathcal{E}^{(i)}$, are denoted by:

$$
\left\{u_{\sigma, i}, \sigma \in \mathcal{E}^{(i)}, 1 \leq i \leq d\right\}
$$

Let us now turn to the treatment of Dirichlet boundary conditions. Let $\mathcal{E}_{D} \subset \mathcal{E}_{\text {ext }}$ be the set of faces located on the Dirichlet boundary, and let $\boldsymbol{u}_{D}$ be the prescribed value of the velocity on these faces. For the Rannacher Turek (RT) or Crouzeix-Raviart (CR) discretizations, as usual in the finite element framework, these Dirichlet boundary conditions are built-in in the definition of the discrete space:

$$
\forall \sigma \in \mathcal{E}_{D}, \text { for } 1 \leq i \leq d, \quad u_{\sigma, i}=\frac{1}{|\sigma|} \int_{\sigma} u_{D, i}
$$

where $u_{D, i}$ stands for the $i^{\text {th }}$ component of $\boldsymbol{u}_{D}$. For the MAC scheme, the normal components of the velocity at the Dirichlet boundary are also prescribed:

$$
\text { for } 1 \leq i \leq d, \forall \sigma \in \mathcal{E}_{D} \cap \mathcal{E}^{(i)}, \quad u_{\sigma, i}=\frac{1}{|\sigma|} \int_{\sigma} u_{D, i},
$$

while Dirichlet conditions for tangential components will be used, as usual for finite volumes, in the definition of the diffusion term.

\subsection{Discrete equations}

We now describe the space discretization of each equation of the time semi-discrete algorithm. We choose to present the equations of the projection step in their original form, i.e. before the derivation of the elliptic problem for the pressure, which is thoroughly described in [10]. Indeed, this latter computation is purely algebraic, in the sense that it transforms a non-linear algebraic system into another non-linear algebraic system which is strictly equivalent, and thus has no impact on the properties of the scheme (besides, of course, the efficiency issue).

We begin with the discretization of the mass balance equations (12b) and (12c) of the projection step. Let $u_{K, \sigma}^{n+1}$ be an approximation of the normal velocity to the face $\sigma$ outward $K$, defined by:

$$
u_{K, \sigma}^{n+1}=\mid \begin{aligned}
& u_{\sigma, i}^{n+1} \boldsymbol{n}_{K, \sigma} \cdot \boldsymbol{e}^{(i)} \text { for } \sigma \in \mathcal{E}^{(i)} \text { in the MAC case, } \\
& \boldsymbol{u}_{\sigma}^{n+1} \cdot \boldsymbol{n}_{K, \sigma} \text { in the } \mathrm{CR} \text { and RT cases. }
\end{aligned}
$$


Pressure correction staggered schemes for barotropic one-phase and two-phase flows

Discrete equations are obtained by an upwind finite volume discretization and read:

$$
\begin{aligned}
\forall K \in \mathcal{M}, \quad \frac{|K|}{\delta t}\left(\rho_{K}^{n+1}-\rho_{K}^{n}\right)+\sum_{\sigma \in \mathcal{E}(K)}|\sigma| u_{K, \sigma}^{n+1} \rho_{\sigma}^{n+1}=0, \\
\frac{|K|}{\delta t}\left(z_{K}^{n+1}-z_{K}^{n}\right)+\sum_{\sigma \in \mathcal{E}(K)}|\sigma| u_{K, \sigma}^{n+1} z_{\sigma}^{n+1}=0,
\end{aligned}
$$

where $\rho_{\sigma}^{n+1}$ (resp. $z_{\sigma}^{n+1}$ ) is the upwind approximation of $\rho^{n+1}$ (resp. $z^{n+1}$ ) at the face $\sigma$, the definition of which we now recall for the sake of completeness. For an internal face $\sigma=K \mid L$, $\rho_{\sigma}^{n+1}\left(\operatorname{resp} . z_{\sigma}^{n+1}\right)$ stands for $\rho_{K}^{n+1}\left(\operatorname{resp} . z_{K}^{n+1}\right)$ if $u_{K, \sigma}^{n+1} \geq 0$ and for $\rho_{L}^{n+1}$ (resp. $z_{L}^{n+1}$ ) otherwise; for an external face $\sigma \in \mathcal{E}(K), \rho_{\sigma}^{n+1}$ (resp. $z_{\sigma}^{n+1}$ ) is equal to $\rho_{K}^{n+1}$ (resp. $z_{K}^{n+1}$ ) if the flow is directed outward $\Omega$ (i.e. $u_{K, \sigma}^{n+1} \geq 0$ ) or given by the boundary conditions otherwise. This approximation ensures that $\rho^{n+1}>0$ if $\rho^{n}>0$ and if the density is prescribed to a positive value at inflow boundaries, without any constraint on the time step. In addition, if we set, for $K \in \mathcal{M}, y_{K}^{n+1}=z_{K}^{n+1} / \rho_{K}^{n+1}$ and $y_{K}^{n}=z_{K}^{n} / \rho_{K}^{n}$, we may recast the second equation of (15) as:

$$
\frac{|K|}{\delta t}\left(\rho_{K}^{n+1} y_{K}^{n+1}-\rho_{K}^{n} y_{K}^{n}\right)+\sum_{\sigma \in \mathcal{E}(K)}|\sigma| \boldsymbol{u}_{\sigma}^{n+1} \cdot \boldsymbol{n}_{K, \sigma} \rho_{\sigma}^{n+1} y_{\sigma}^{n+1}=0,
$$

where we recognize in $y_{\sigma}^{n+1}$ the upwind approximation of $y^{n+1}$ at the face $\sigma$. This relation thus yields that $y^{n+1}$ satisfies a discrete maximum principle by standard arguments [21].
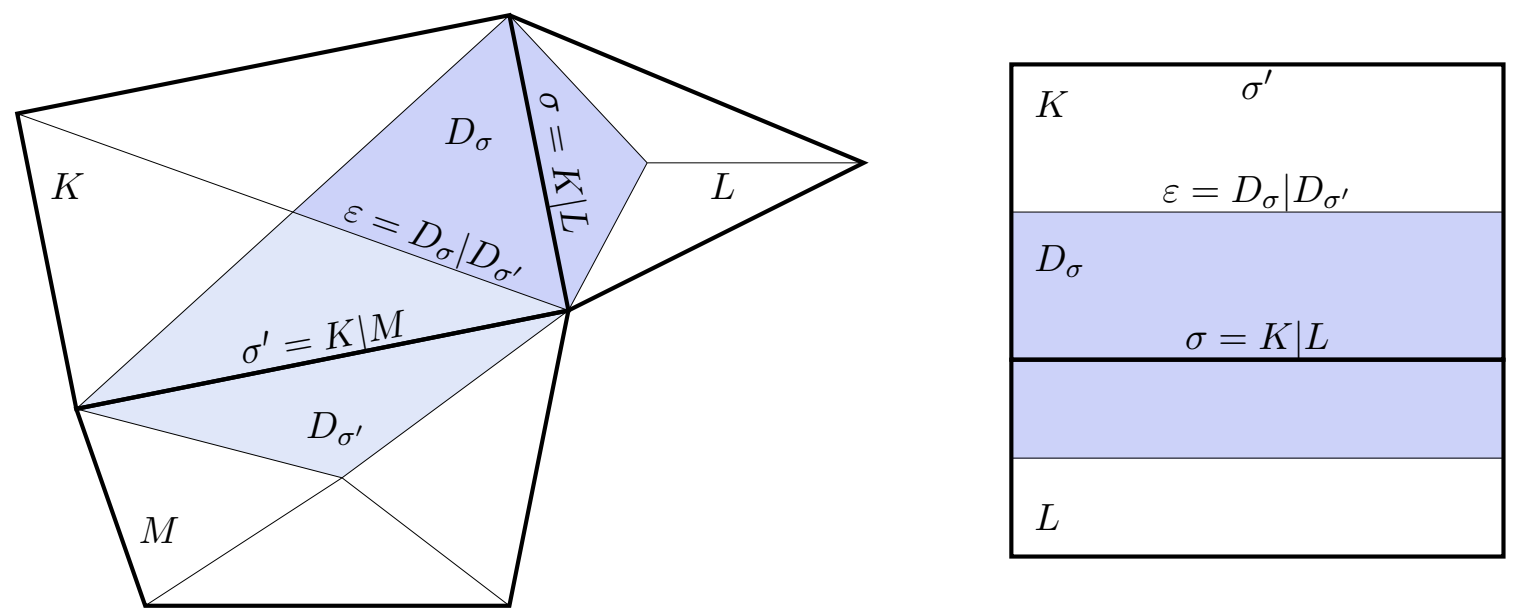

Figure 1: Notations for control volumes and dual cells - Left: finite elements (the present sketch illustrates the possibility, implemented in our software (ISIS [19]), of mixing simplicial (Crouzeix-Raviart) and quadrangular (Rannacher-Turek) cells) - Right: MAC discretization, dual cell for the $y$-component of the velocity.

In the case of the MAC discretization, the velocity prediction equation is approximated by a finite volume technique over a dual mesh. For the RT and CR discretizations, the time derivative and convection terms are approximated by a similar finite volume technique, while the finite element formulation is used for the other terms. For each component of the velocity, a dual mesh of the computational domain $\Omega$ thus has to be defined: 
Pressure correction staggered schemes for barotropic one-phase and two-phase flows

- Rannacher-Turek or Crouzeix-Raviart discretizations - For the RT or CR discretizations, the dual mesh is the same for all the velocity components. When $K \in \mathcal{M}$ is a simplex, a rectangle or a cuboid, for $\sigma \in \mathcal{E}(K)$, we define $D_{K, \sigma}$ as the cone with basis $\sigma$ and with vertex the mass center of $K$ (see Figure 1). We thus obtain a partition of $K$ in $m$ sub-volumes, where $m$ is the number of faces of the mesh, each sub-volume having the same measure $\left|D_{K, \sigma}\right|=|K| / m$. We extend this definition to general quadrangles and hexahedra, by supposing that we have built a partition still of equal-volume sub-cells, and with the same connectivities; note that this is of course always possible, but that such a volume $D_{K, \sigma}$ may be no longer a cone; indeed, if $K$ is far from a parallelogram, it may not be possible to build a cone having $\sigma$ as basis, the opposite vertex lying in $K$ and a volume equal to $|K| / m$. The volume $D_{K, \sigma}$ is referred to as the half-diamond cell associated to $K$ and $\sigma$.

For $\sigma \in \mathcal{E}_{\text {int }}, \sigma=K \mid L$, we now define the diamond cell $D_{\sigma}$ associated to $\sigma$ by $D_{\sigma}=$ $D_{K, \sigma} \cup D_{L, \sigma}$; for an external face $\sigma \in \mathcal{E}_{\text {ext }} \cap \mathcal{E}(K), D_{\sigma}$ is just the same volume as $D_{K, \sigma}$.

- MAC discretization - For the MAC scheme, the dual mesh depends on the component of the velocity. For each component, the MAC dual mesh only differs from the RT or CR dual mesh by the choice of the half-diamond cell, which, for $K \in \mathcal{M}$ and $\sigma \in \mathcal{E}(K)$, is now the rectangle or rectangular parallelepiped of basis $\sigma$ and of measure $\left|D_{K, \sigma}\right|=|K| / 2$.

We denote by $\left|D_{\sigma}\right|$ the measure of the dual cell $D_{\sigma}$, and by $\varepsilon=D_{\sigma} \mid D_{\sigma^{\prime}}$ the face separating two diamond cells $D_{\sigma}$ and $D_{\sigma^{\prime}}$ (see Figure 1).

In both cases, for $1 \leq i \leq d$ and $\sigma \in \mathcal{E}^{(i)}$, we denote by $(\operatorname{div} \tau(\tilde{\boldsymbol{u}}))_{\sigma, i}^{n+1}$ an approximation of the $i$ th component of the viscous term associated to $\sigma$, and we denote by $(\nabla p)_{\sigma, i}^{n}$ the $i$-th component of the discrete pressure gradient at the face $\sigma$. With these notations, we are able to write the following general form of the approximation to the momentum balance equation:

$$
\begin{aligned}
\frac{\left|D_{\sigma}\right|}{\delta t}\left(\rho_{D_{\sigma}}^{n} \tilde{u}_{\sigma, i}^{n+1}-\rho_{D_{\sigma}}^{n-1} u_{\sigma, i}^{n}\right) & +\sum_{\varepsilon \in \mathcal{E}\left(D_{\sigma}\right)} F_{\sigma, \varepsilon}^{n} \tilde{u}_{\varepsilon, i}^{n+1} \\
& +\left|D_{\sigma}\right|(\nabla p)_{\sigma, i}^{n}-\left|D_{\sigma}\right|(\operatorname{div} \tau(\tilde{\boldsymbol{u}}))_{\sigma, i}^{n+1}=f_{\sigma, i}^{n+1},
\end{aligned}
$$

this equation being written for $1 \leq i \leq d, \sigma \in \mathcal{E} \backslash \mathcal{E}_{D}$ in the case of the RT or CR discretizations, and for $1 \leq i \leq d, \sigma \in \mathcal{E}^{(i)} \backslash \mathcal{E}_{D}$ for the MAC scheme. In this relation, $\rho_{D_{\sigma}}^{n}$ and $\rho_{D_{\sigma}}^{n-1}$ stand for an approximation of the density on the face $\sigma$ at time $t^{n}$ and $t^{n-1}$ respectively (which must not be confused with the upstream density $\rho_{\sigma}^{n}$ used in the mass balance), $F_{\sigma, \varepsilon}^{n}$ is the discrete mass flux through the dual face $\varepsilon$ outward $D_{\sigma}$ (defined later), and $\tilde{u}_{\varepsilon, i}^{n+1}$ stands for an approximation of $\tilde{\boldsymbol{u}}_{i}^{n+1}$ on $\varepsilon$ which may be chosen either centred or upwind. In the centred case, for an interior face $\varepsilon=D_{\sigma} \mid D_{\sigma^{\prime}}$, we thus get $\tilde{u}_{\varepsilon, i}^{n+1}=\left(\tilde{u}_{\sigma, i}^{n+1}+\tilde{u}_{\sigma^{\prime}, i}^{n+1}\right) / 2$ while, in the upwind case, we have $\tilde{u}_{\varepsilon, i}^{n+1}=\tilde{u}_{\sigma, i}^{n+1}$ if $F_{\sigma, \varepsilon}^{n} \geq 0$ and $\tilde{u}_{\varepsilon, i}^{n+1}=\tilde{u}_{\sigma^{\prime}, i}^{n+1}$ otherwise.

The quantity $f_{\sigma, i}^{n+1}$ is a forcing term, which, for our purpose here, does not vanish only on external faces where Neumann conditions are prescribed; if these latter read $\boldsymbol{\tau} \cdot \boldsymbol{n}-p \boldsymbol{n}=\boldsymbol{f}$, we get:

$$
f_{\sigma, i}^{n+1}=\frac{1}{\delta t} \int_{n \delta t}^{(n+1) \delta t} \int_{\sigma} \boldsymbol{f} \cdot \boldsymbol{e}^{(i)} \mathrm{d} \gamma
$$

The finite element discretization of the $i$-th component of the pressure gradient term reads:

$$
\left|D_{\sigma}\right|(\nabla p)_{\sigma, i}^{n}=-\sum_{K \in \mathcal{M}} \int_{K} p^{n} \operatorname{div} \varphi_{\sigma}^{(i)} \mathrm{d} \boldsymbol{x} .
$$


Pressure correction staggered schemes for barotropic one-phase and two-phase flows

Since the pressure is piecewise constant, using the definition of the RT or CR shape functions, an easy computation yields for an internal face $\sigma=K \mid L$ :

$$
\left|D_{\sigma}\right|(\nabla p)_{\sigma, i}^{n}=|\sigma|\left(p_{L}^{n}-p_{K}^{n}\right) \boldsymbol{n}_{K, \sigma} \cdot \boldsymbol{e}^{(i)},
$$

and, for an external face $\sigma \in \mathcal{E}(K) \cap \mathcal{E}_{\text {ext }} \backslash \mathcal{E}_{D}$ :

$$
\left|D_{\sigma}\right|(\nabla p)_{\sigma, i}^{n}=-|\sigma| p_{K}^{n} \boldsymbol{n}_{K, \sigma} \cdot \boldsymbol{e}^{(i)} .
$$

These expressions coincide which the discrete gradient in the MAC discretization.

The finite element discretization of the viscous term $(\operatorname{div} \tau(\tilde{\boldsymbol{u}}))_{\sigma, i}^{n+1}$, associated to $\sigma$ and to the component $i$, reads:

$$
\left|D_{\sigma}\right|(\operatorname{div} \tau(\tilde{\boldsymbol{u}}))_{\sigma, i}^{n+1}=-\mu \sum_{K \in \mathcal{M}} \int_{K} \nabla \tilde{\boldsymbol{u}}^{n+1} \cdot \nabla \boldsymbol{\varphi}_{\sigma}^{(i)}-\frac{\mu}{3} \sum_{K \in \mathcal{M}} \int_{K} \operatorname{div} \tilde{\boldsymbol{u}}^{n+1} \operatorname{div} \boldsymbol{\varphi}_{\sigma}^{(i)} .
$$

The MAC discretization of this same viscous term is detailed in [3].

The main motivation to implement a finite volume approximation for the first two terms is to obtain a discrete equivalent of the kinetic energy theorem, which reads, for instance in the case of homogeneous Dirichlet boundary conditions:

$$
\begin{aligned}
\sum_{i=1}^{d} \sum_{\sigma \in \mathcal{E}_{\text {int }}^{(i)}}\left[\frac{\left|D_{\sigma}\right|}{\delta t}\left(\rho_{D_{\sigma}}^{n} \tilde{u}_{\sigma, i}^{n+1}-\rho_{D_{\sigma}}^{n-1} u_{\sigma, i}^{n}\right)+\sum_{\varepsilon \in \mathcal{E}\left(D_{\sigma}\right)} F_{\sigma, \varepsilon}^{n} \tilde{u}_{\varepsilon, i}^{n+1}\right] \tilde{u}_{\sigma, i} \geq \\
\frac{1}{2} \sum_{i=1}^{d} \sum_{\sigma \in \mathcal{E}_{\text {int }}^{(i)}} \frac{\left|D_{\sigma}\right|}{\delta t}\left[\rho_{D_{\sigma}}^{n}\left(\tilde{u}_{\sigma, i}^{n+1}\right)^{2}-\rho_{D_{\sigma}}^{n-1}\left(u_{\sigma, i}^{n}\right)^{2}\right] .
\end{aligned}
$$

For this result to be valid, the necessary condition is that the convection operator vanishes for a constant velocity, i.e. that the following discrete mass balance over the diamond cells is satisfied $[1,8]$ :

$$
\forall \sigma \in \mathcal{E}_{\text {int }}, \quad \frac{\left|D_{\sigma}\right|}{\delta t}\left(\rho_{D_{\sigma}}^{n}-\rho_{D_{\sigma}}^{n-1}\right)+\sum_{\varepsilon \in \mathcal{E}\left(D_{\sigma}\right)} F_{\sigma, \varepsilon}^{n}=0 .
$$

This governs the choice for the definition of the density approximation $\rho_{D_{\sigma}}$ and the mass fluxes $F_{\sigma, \varepsilon}$. The density $\rho_{D_{\sigma}}$ is defined by a weighted average:

$$
\forall \sigma \in \mathcal{E}_{\text {int }}, \sigma=K|L,| D_{\sigma}\left|\rho_{D_{\sigma}}=\right| D_{K, \sigma}\left|\rho_{K}+\right| D_{L, \sigma} \mid \rho_{L}
$$

and, $\forall \sigma \in \mathcal{E}_{\text {ext }} \backslash \mathcal{E}_{D}, \sigma \in \mathcal{E}(K), \rho_{D_{\sigma}}=\rho_{K}$. For the MAC scheme, the flux on a dual face which is located on two primal faces is the mean value of the sum of fluxes on the two primal faces, and the flux of a dual face located between two primal faces is again the mean value of the sum of fluxes on the two primal faces [18]. In the case of the CR and RT schemes, for a dual face $\varepsilon$ included in the primal cell $K$, this flux is computed as a linear combination (with constant coefficients, i.e. independent of the cell) of the mass fluxes through the faces of $K$, i.e. the quantities $\left(|\sigma| u_{K, \sigma}^{n+1} \rho_{\sigma}^{n+1}\right)_{\sigma \in \mathcal{E}(K)}$ appearing in the discrete mass balance (first relation of (15)). We refer to $[1,9]$ for a detailed construction of this approximation.

Equation (12a) is discretized similarly to the momentum balance (17), i.e. a finite volume technique is used for the time derivative term in the RT and CR discretizations. Hence, for both schemes, the discretization of (12a) reads:

$$
\frac{\left|D_{\sigma}\right|}{\delta t} \rho_{D_{\sigma}}^{n}\left(\boldsymbol{u}_{\sigma, i}^{n+1}-\tilde{\boldsymbol{u}}_{\sigma, i}^{n+1}\right)+\left|D_{\sigma}\right|\left[(\boldsymbol{\nabla} p)_{\sigma, i}^{n+1}-(\boldsymbol{\nabla} p)_{\sigma, i}^{n}\right]=0
$$


Pressure correction staggered schemes for barotropic one-phase and two-phase flows

this equation being written for $1 \leq i \leq d, \sigma \in \mathcal{E} \backslash \mathcal{E}_{D}$ in the case of the RT and CR discretizations, and for $1 \leq i \leq d, \sigma \in \mathcal{E}^{(i)} \backslash \mathcal{E}_{D}$ for the MAC scheme.

\section{Riemann problems}

In this section, we assess the behaviour of the scheme for several 1D Riemann problems (often called also "shock tube problems") for the hyperbolic system (1)-(3) with $\mu=0$ in (4), for which an analytical expression of the solution is known and which have been extensively studied numerically (see e.g. [11]). We have seen that the pressure correction scheme is able to keep $y=1$ at any time, if the initial and boundary conditions allow; we may therefore first address a single phase flow, namely the solution of the so-called "Sod shock tube" problem (Section 3.1) before turning to two-phase flows, namely "two-fluid shock tube" model problems (Section 3.2).

The computations presented here are performed with the ISIS code [19], built from the software component library PELICANS [24], both under development at IRSN and available as opensource softwares. The ISIS computer code is devoted to the solution of $2 \mathrm{D}$ or $3 \mathrm{D}$ problems (as the scheme presented in previous sections), so we are led to define a fake 2D problem, designed to boil down to the addressed 1D Riemann problem. The domain $\Omega$ is rectangular, and the mesh is composed of only one horizontal stripe of cells (see Figures $2-4$ ).

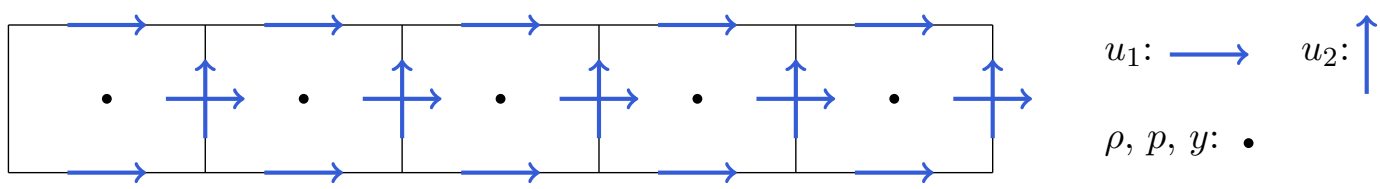

Figure 2: Primal mesh and location of the unknowns for the Rannacher-Turek element, for a mesh consisting of only one stripe of cells, with symmetry (i.e. perfect slip) conditions at the top and bottom boundaries, Neumann conditions at the right boundary, and Dirichlet boundary conditions at the left boundary.

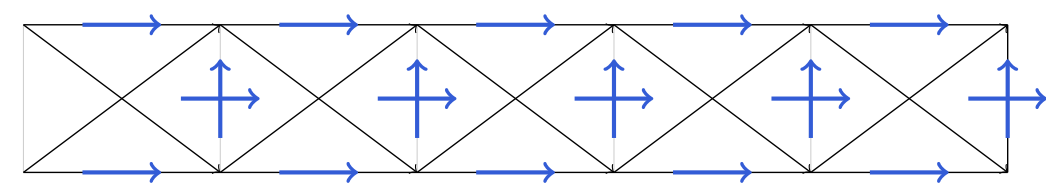

Figure 3: Dual finite volume mesh for the Rannacher-Turek element, with the same boundary conditions as in Figure 2.

In order to define a one-dimensional problem on this two dimensional domain, we impose a symmetry condition to the velocity at the top and bottom of the domain $\Omega$ (i.e., with $\boldsymbol{u}=$ $\left(u_{1}, u_{2}\right)$, we set $u_{2}=0$ and $\left.\partial_{x_{2}} u_{1}=0\right)$, which is satisfied by an horizontal flow invariant with respect to the second coordinate. An easy computation shows that, with the chosen mesh and boundary conditions, we obtain a discrete problem which exactly coincides with a 1D discretization for the MAC scheme; this is clearly not the case for the RT element, since degrees of freedom for the horizontal velocity subsist at three different vertical locations. 
Pressure correction staggered schemes for barotropic one-phase and two-phase flows



Figure 4: Primal mesh and location of the unknowns for the MAC discretization, for a mesh consisting of only one stripe of cells, with the same boundary conditions as in Figure 2.

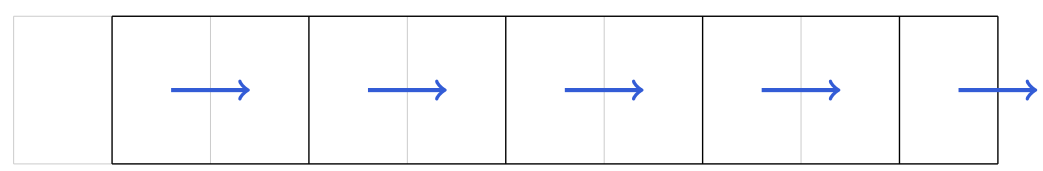

Figure 5: Dual finite volume mesh for the MAC discretization, for a mesh consisting in only one stripe of meshes, with the same boundary conditions as in Figure 2.

All the solutions computed in the following are such that the flow enters the domain on the left and leaves it on the right. So, at the left side of the domain, we impose $\boldsymbol{u}=\left(u_{L}, 0\right), \rho=\rho_{L}$ and $z=z_{L}$; at the right hand side of the domain, we prescribe a non-homogeneous Neumann boundary condition, with a surface forcing term equal to $-p_{R} \boldsymbol{n}$, where $\boldsymbol{n}$ is the unit outward normal vector to the boundary $\partial \Omega$.

As described above, for the velocity convection term in the momentum balance equation, the approximation of the velocity at the faces of the dual mesh (see Figures 3 and 5) may be chosen centred or upwind; we will refer to the first option in the following as the centred variant (although upwinding is always used in the discrete mass balance equations), and to the second one as the upwind variant.

\subsection{Sod shock tube problem}

In order to simulate the Sod shock tube test, the gas mass fraction is set to $y \equiv 1$ (onephase problem); we consider here the non-viscous homogeneous model resulting from Equations (1)-(3), with $\mu=0$, with the equation of state $p=\rho$, which corresponds to the isothermal Euler equations. The (1D) continuous problem is posed over the interval $(-2,3)$ and, for the computation, we take $\Omega=(-2,3) \times(0,0.01)$. The two initial constant states are given by:

$$
\left(\begin{array}{l}
\rho \\
u
\end{array}\right)_{L}=\left(\begin{array}{l}
1 \\
0
\end{array}\right), \quad\left(\begin{array}{l}
\rho \\
u
\end{array}\right)_{R}=\left(\begin{array}{l}
0.125 \\
0
\end{array}\right) .
$$

With this initial condition, the solution consists in a rarefaction wave traveling to the left and a shock traveling to the right.

We first address the results obtained with the RT discretization. The first outcome is that the scheme converges to the exact solution as soon as some diffusion is introduced in the momentum balance equation, either by adding a small artificial viscosity term to the centred approximation or by using the upwind scheme; for $\mu=0$ and the centred variant, the usual (for a centred discretization of the advection operator) odd-even oscillations affect the computed velocity, and 
Pressure correction staggered schemes for barotropic one-phase and two-phase flows

convergence is lost. More precisely, due to the particular structure of the mesh (see Figures 2 and 3 ), we observe in this latter case that the solution seems to result from the superposition of two different regular functions, one associated to the degrees of freedom located at the intermediate elevation and the second one associated to the degrees of freedom located on the top and bottom boundaries; surprisingly, these two functions do not change when refining the mesh and time step at constant CFL number. As an example of the numerical results obtained in convergent cases, the solution at $t=1$ obtained with a mesh consisting of 2000 cells, $\delta t=0.00125$ and a residual viscosity of $\mu=0.001$ is presented in Figure 6, together with the exact solution. Using $v=1.6$ (which corresponds approximately to the velocity of the faster wave, namely the shock) as velocity range, these numerical parameters correspond to a CFL $=v \delta t / h=0.8$.

Since combining a centred discretization of the momentum balance equation with the addition of an artificial viscosity may seem to be an appealing technique to avoid an excessive numerical dissipation (for instance, associated to an adjustment of $\mu$ as a function of the regularity of the solution, in the spirit of $[12,13])$, we now investigate the influence of the value of $\mu$ on the accuracy of the centred scheme. We observe in Figure 7 and Figure 8 that taking a large viscosity yields inaccurate results, which is easily explained by the fact that the solved problem is too far from the original one. On the other hand, for too low values of the viscosity, oscillations appear, and the numerical error increases. In between, the error remains small, and one can remark that the optimal value for $\mu$ with respect to the $L^{1}$ norm of the error decreases with the time and space steps, as would also be the case for the numerical dissipation introduced by the upwinding technique. In addition, the optimal viscosity seems to be significantly smaller than the artificial dissipation introduced by the upwinding (i.e. $\rho u h / 2$ ). Comparing Figures 7 and 8 , we note that the plateau is wider for $\mathrm{CFL}=9.6$ than for $\mathrm{CFL}=0.8$, but the overall shape of the curves remains essentially similar for both CFL numbers.

We end this study of the RT discretization by reporting the accuracy of the schemes as a function of the time and space step, with two constant CFL numbers. We study the centred scheme with $\mu=0.001$ and the upwind scheme with $\mu=0$ (we shall always set $\mu=0$ for the upwind scheme hereafter). For the centred scheme, the observed orders of convergence (Figure 9) are about 0.5 at $\mathrm{CFL}=0.8$ and 1 at $\mathrm{CFL}=9.6$ respectively, for both the velocity and the pressure. For the upwind variant, the order of convergence is 0.75 for any CFL.

With the MAC discretization, the behaviour is quite different, since the scheme seems to be convergent in both its velocity-centred and upwind version, without any additional artificial viscosity. The solution at $t=1$ obtained with the same parameters as for the RT discretization (i.e. 2000 cells, $\delta t=0.00125$, so $\mathrm{CFL}=0.8$, and a residual viscosity of $\mu=0.001$ ) is presented in Figure 10. The influence of the addition of an artificial viscosity to the centred variant is shown in Figures 11 and 12. Finally, we once again assess the accuracy of the schemes as a function of the time and space step, keeping a constant CFL for two different numbers (Figure 13); all the computations are now performed with $\mu=0$. Results seem to indicate that the order of convergence does not depend on the CFL number, nor on the upwind or centred choice: in all cases, the order of convergence is close to 0.8 , for the velocity and the pressure; note that the convergence is reported to 1 for a collocated upwind scheme [2]. 

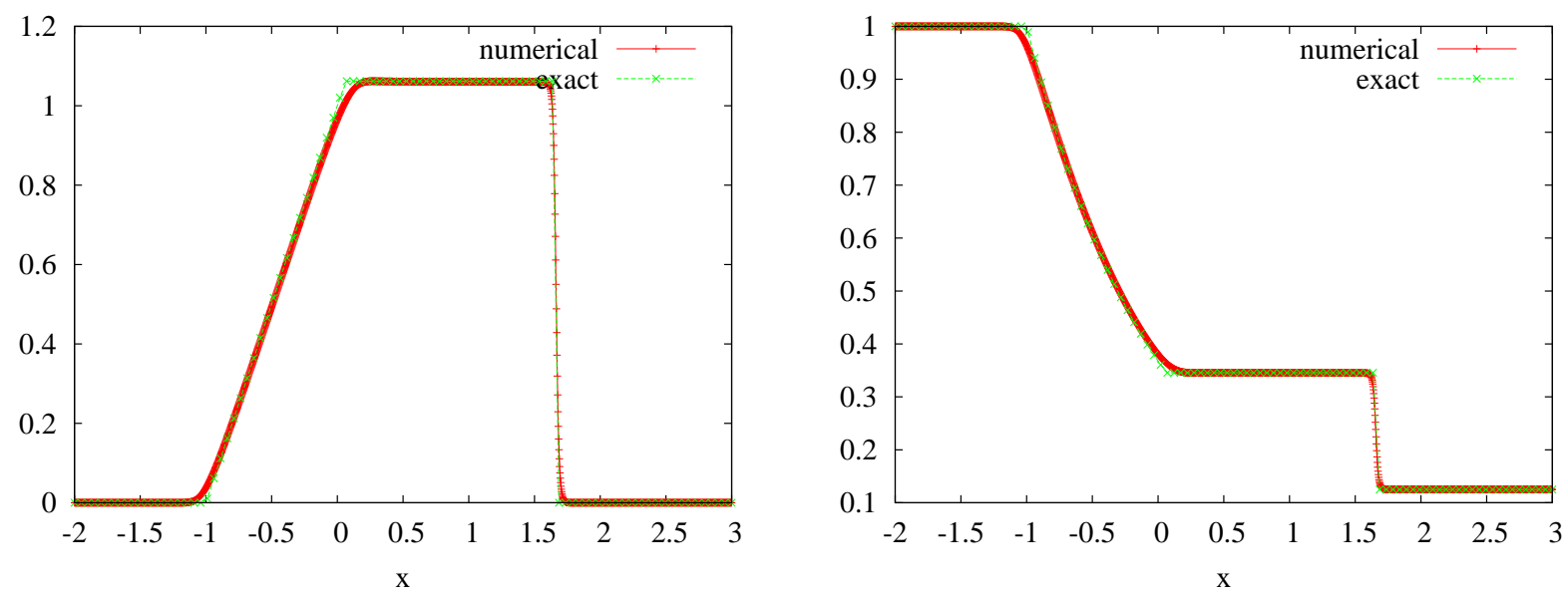

Figure 6: Sod shock tube problem - Centred RT scheme - Numerical solution of the perturbed viscous problem at $t=1$ with $\mu=0.001,2000$ cells, $\delta t=0.00125$ (i.e. $\mathrm{CFL}=0.8$ ). Velocity (left) and pressure (right).
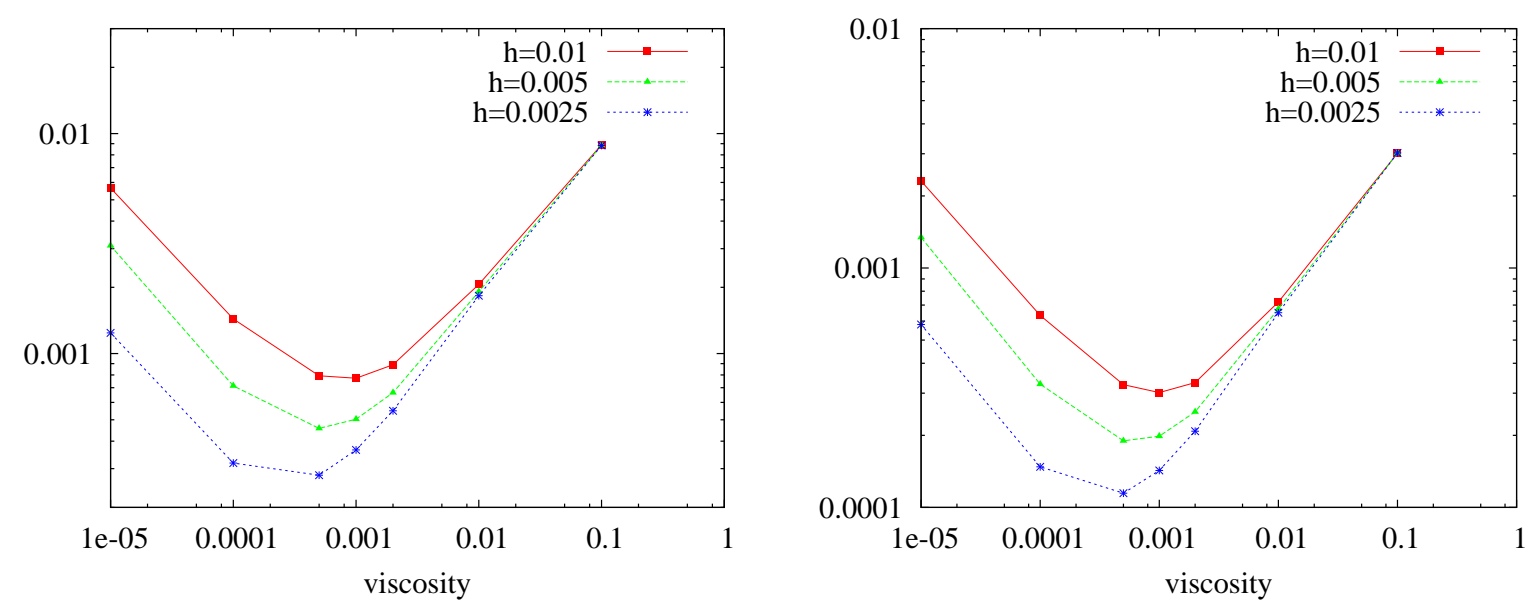

Figure 7: Sod shock tube problem - Centred RT scheme $-L^{1}$ norm of the error between numerical solutions of the perturbed viscous problem and the exact solution of the inviscid problem at $t=1$, for three meshes, as a function of the viscosity $\mu$, with $\mathrm{CFL}=0.8$. Velocity (left) and pressure (right). 

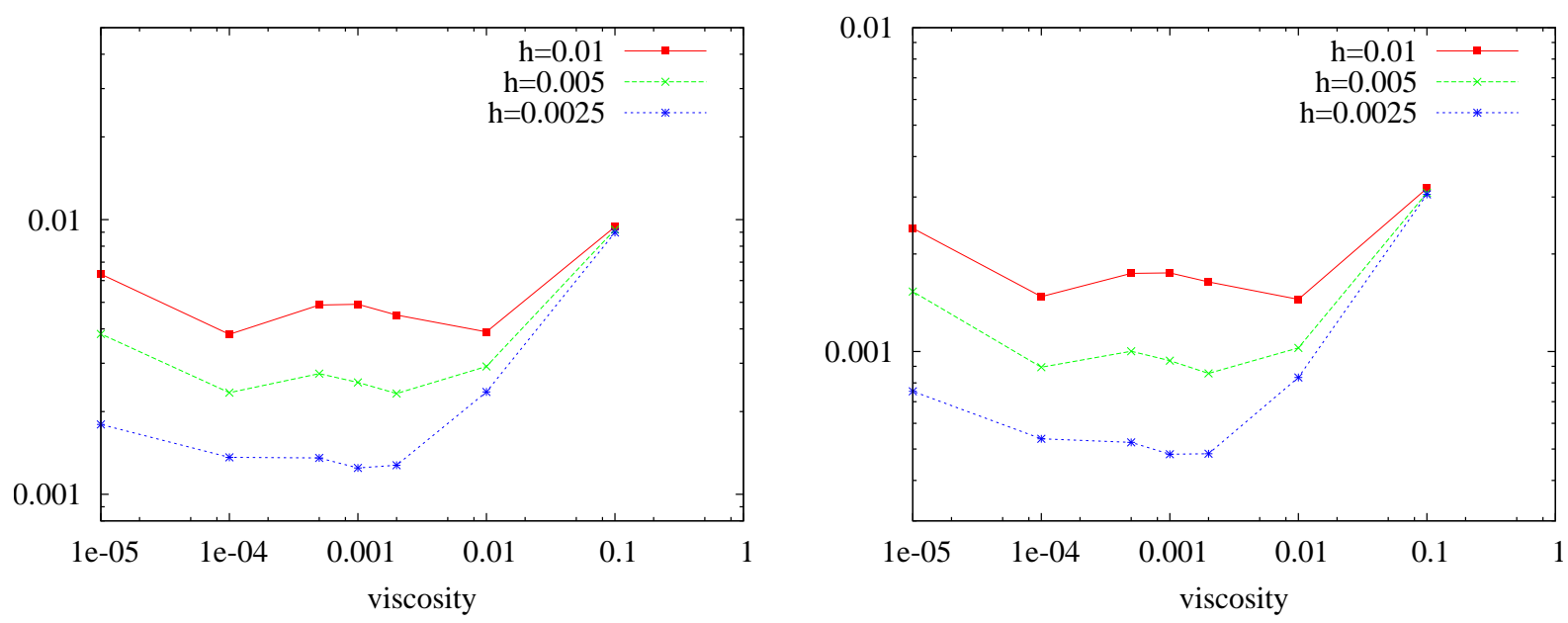

Figure 8: Sod shock tube problem - Centred RT scheme $-L^{1}$ norm of the error between numerical solutions of the perturbed viscous problem and the exact solution of the inviscid problem at $t=1$, for three meshes, as a function of the viscosity $\mu$, with $\mathrm{CFL}=9.6$. Velocity (left) and pressure (right).
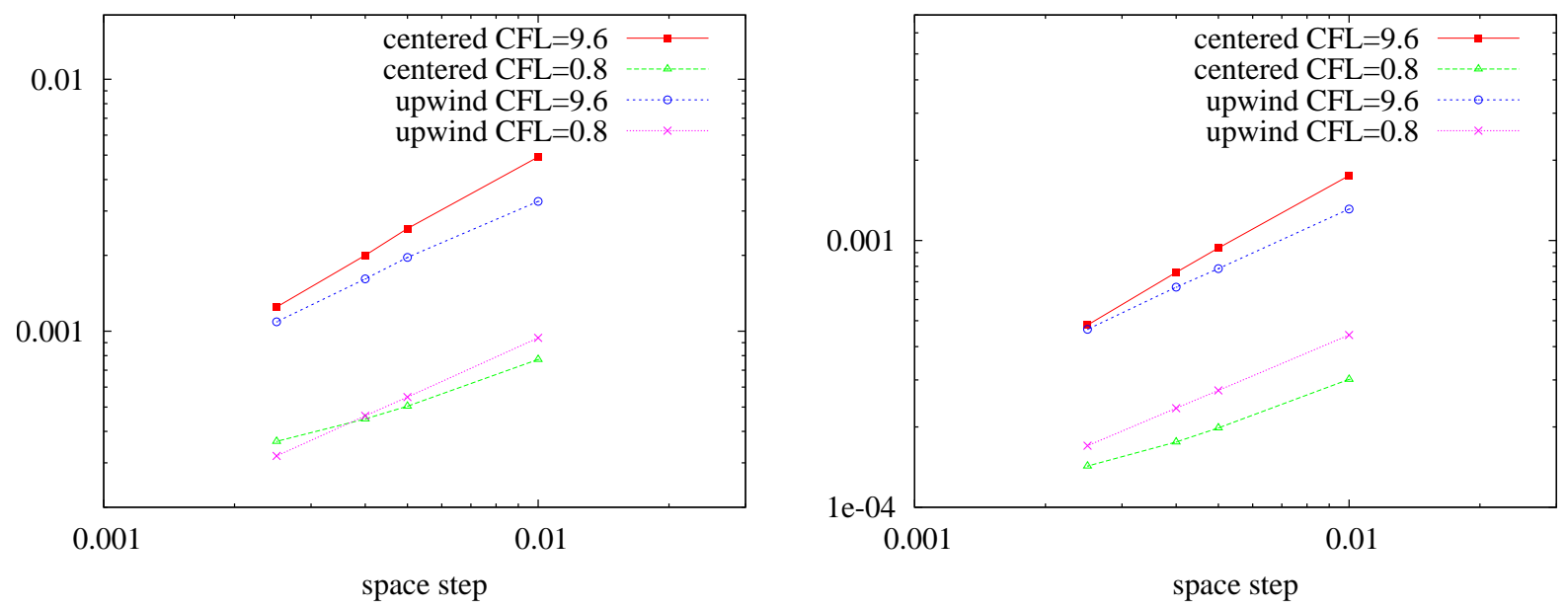

Figure 9: Sod shock tube problem - Centred and upwind RT schemes $-L^{1}$ norm of the error between numerical solutions of the perturbed viscous problem and the exact solution of the inviscid problem at $t=1$, as a function of the mesh (or time) step, for two fixed CFL numbers. In the centred case, the used artificial viscosity is $\mu=0.001$, i.e. a value close to the one which yields the most accurate results. Velocity (left) and pressure (right). 

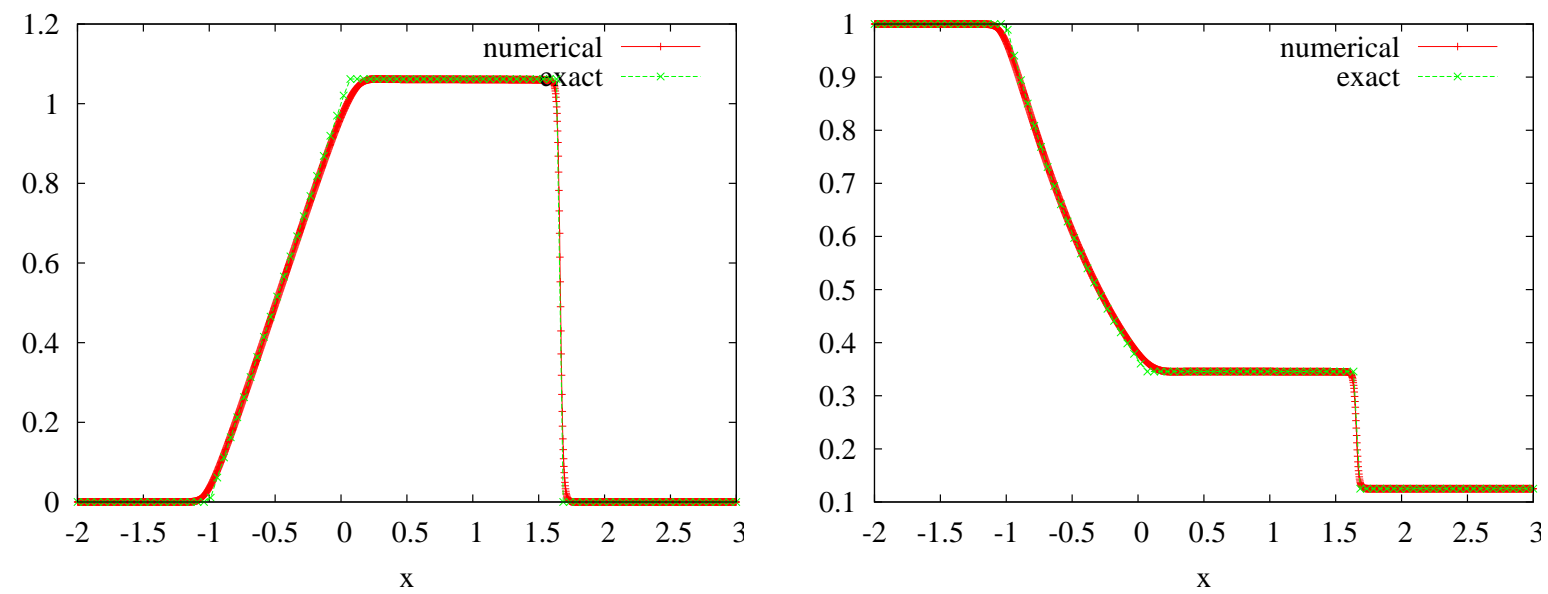

Figure 10: Sod shock tube problem - Centred MAC scheme - Numerical solution of the perturbed viscous problem at $t=1$ with $\mu=0.001,2000$ cells, $\delta t=0.00125$ (i.e. CFL=0.8). Velocity (left) and pressure (right).
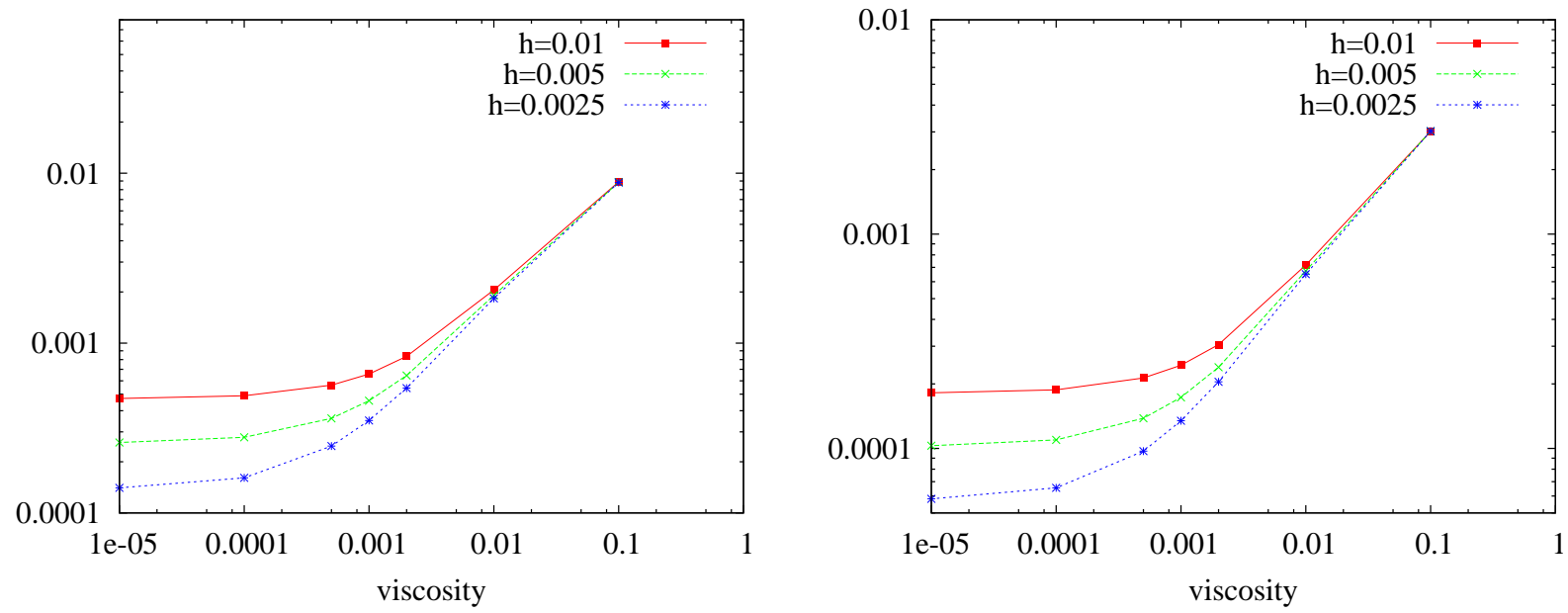

Figure 11: Sod shock tube problem - Centred MAC scheme $-L^{1}$ norm of the error between numerical solutions of the perturbed viscous problem and the exact solution of the inviscid problem at $t=1$, for three meshes, as a function of the viscosity $\mu$, with $\mathrm{CFL}=0.8$. Velocity (left) and pressure (right). 

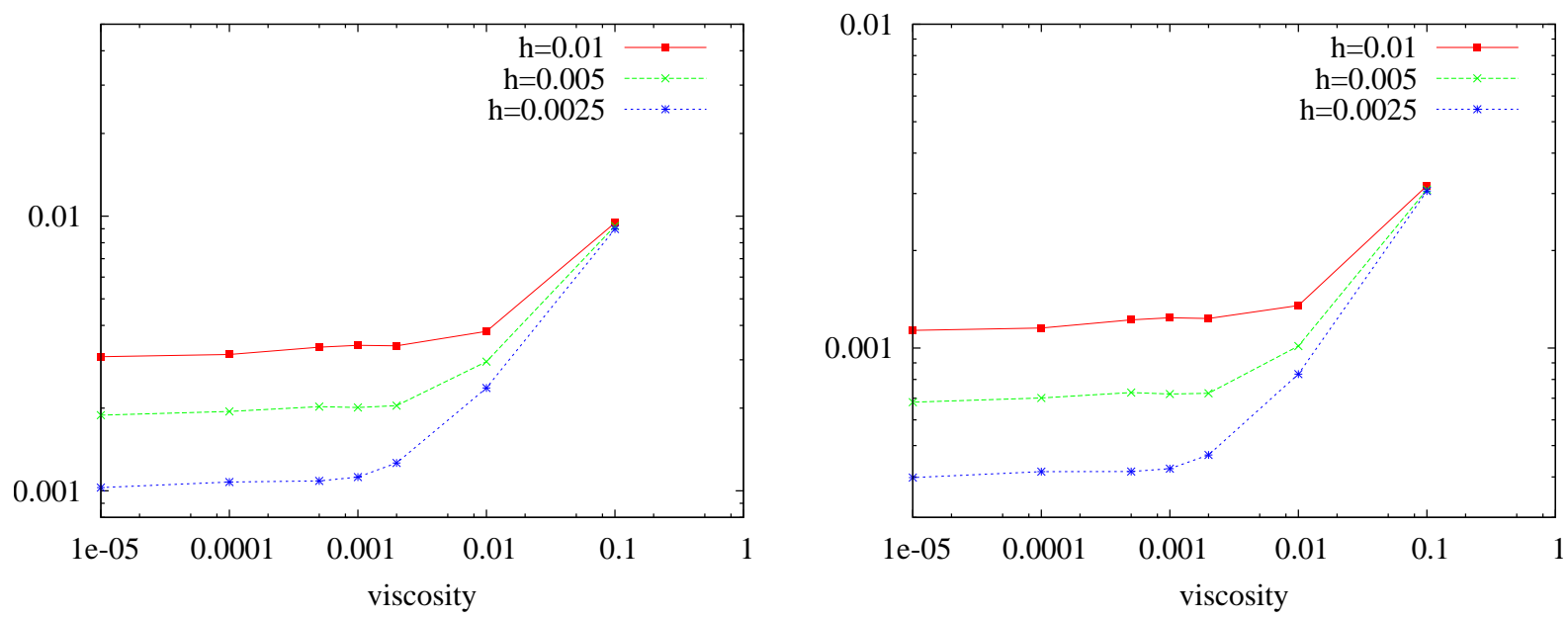

Figure 12: Sod shock tube problem - Centred MAC scheme $-L^{1}$ norm of the error between numerical solutions of the perturbed viscous problem and the exact solution of the inviscid problem at $t=1$, for three meshes, as a function of the viscosity $\mu$, with $\mathrm{CFL}=9.6$. Velocity (left) and pressure (right).
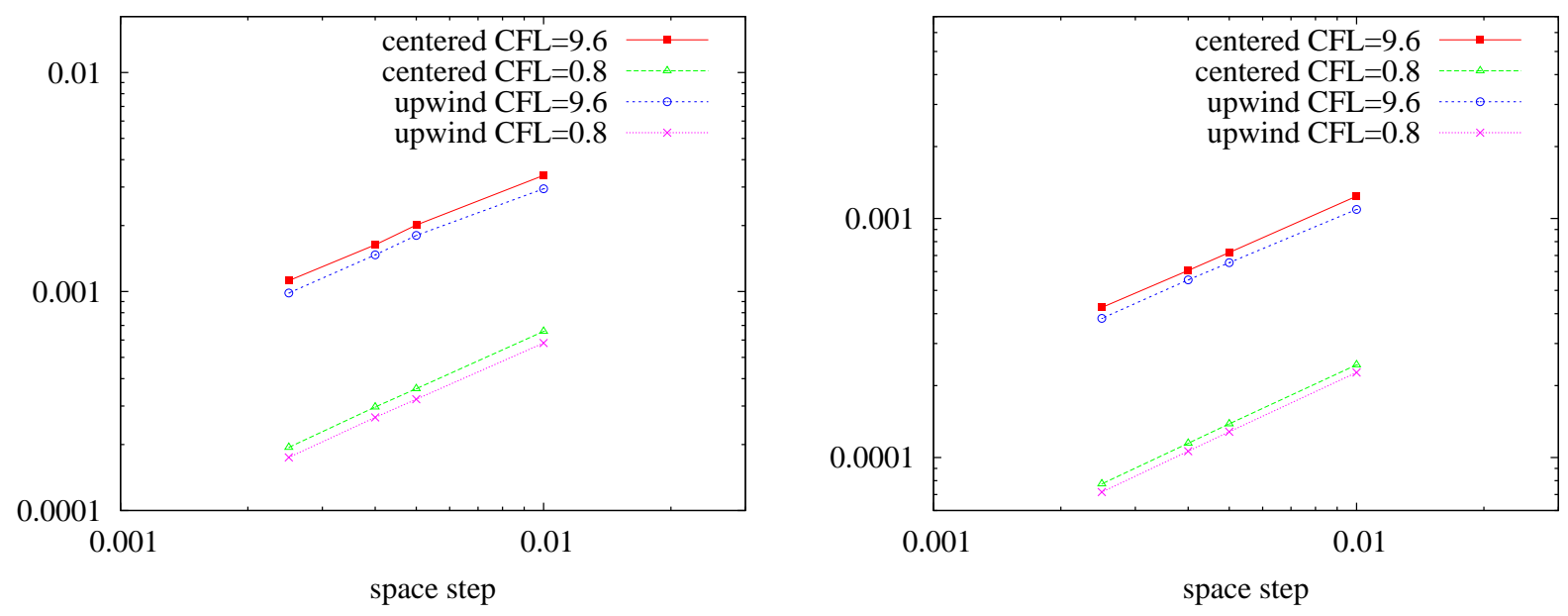

Figure 13: Sod shock tube problem - Centred and upwind MAC scheme $-L^{1}$ norm of the error between the numerical solution and the exact solution at $t=1$, as a function of the mesh (or time) step, for two fixed CFL numbers. Velocity (left) and pressure (right). 
Pressure correction staggered schemes for barotropic one-phase and two-phase flows

\subsection{Two-fluid shock tube}

We present here the numerical results for two-fluid shock tube problems. The continuous problem is posed over $(-3,2)$ and we use a computational rectangular domain $\Omega=(-3,2) \times(0,0.01)$. The equation of state is given by (7), with the following phasic equation of state for the gas phase:

$$
p=10 \rho_{g}
$$

The constant liquid density is set to $\rho_{\ell}=0.8$. We perform two tests, where the initial left and right constant states are chosen in order to yield two different flow structures: a contact discontinuity (in both cases), propagating between two shock waves in the first case, and between two rarefaction waves in the second one.

\subsubsection{First case: shock - contact discontinuity - shock}

The two initial constant states are given by:

$$
\left(\begin{array}{l}
\rho \\
\boldsymbol{u} \\
y
\end{array}\right)_{L}=\left(\begin{array}{l}
1 \\
5 \\
0.3
\end{array}\right), \quad\left(\begin{array}{l}
\rho \\
\boldsymbol{u} \\
y
\end{array}\right)_{R}=\left(\begin{array}{l}
2 \\
1 \\
0.8
\end{array}\right) .
$$

With this initial data, the exact solution consists in two shocks, the first one traveling to the left and the second one to the right, separated by a contact discontinuity slowly moving to the right.

The convergence behaviour of the schemes is quite similar to that of the one-phase case, namely convergence of the upwind scheme or of the centred scheme with a residual viscosity in both cases and non-convergence of the centred scheme with $\mu=0$ and the RT element. A numerical solution given by the centred scheme at $t=0.1$ with 5000 cells, $\delta t=4.10^{-5}$ and $\mu=0.002$ is plotted in Figure 14 and Figure 15 for the RT and MAC discretization respectively, together with the exact solution. Taking $v=18.16$ (the velocity of the fastest wave, namely the right shock), the CFL number for these numerical parameters is $\mathrm{CFL}=v \delta t / h=0.75$.

We then plot the solution obtained at $t=0.1$ for various CFL numbers, with the centred schemes, 2500 cells and $\mu=0.002$. We observe in Figure 16, that with the RT discretization, the solution is qualitatively correct up to a CFL of the order of 20 , and then strongly deteriorates, showing in particular wild velocity and pressure oscillations at the contact discontinuity. On the contrary, we observe more reasonable profiles for the MAC discretization in Figure 17: the oscillations only affect the pressure in the vicinity of the contact discontinuity for large CFL numbers $(\geq 80)$. Note that, in any case, the structure of the solution seems to remain correct, i.e. we do not observe the apparition of spurious waves (for instance, non-entropic shocks), as often happens with a scheme without any numerical diffusion.

We then assess the accuracy of the scheme as a function of the time and space steps, with two constant CFL numbers, for the centred variants with $\mu=0.002$ and for the upwind variants. The observed orders of convergence for the centred RT scheme (Figure 18) are about 1.5 and 1. at $\mathrm{CFL}=0.75$ and 9 respectively, for both the velocity and the pressure; for $\rho$ and $y$, the order of convergence is 0.7 and 0.5 respectively, for both CFL numbers. For the upwind RT scheme, the order of convergence is 1 for both the velocity and the pressure and 0.5 for $\rho$ and $y$, at any CFL number. Again, for the MAC scheme (Figure 19), the order of convergence does not seem to depend on the CFL number nor on the upwind or centred choice: in all cases, the order of convergence is 1 for both the velocity and the pressure and 0.5 for the density and the gas mass 
Pressure correction staggered schemes for barotropic one-phase and two-phase flows

fraction. This behaviour is consistent with what is usually observed with finite volume collocated upwinding schemes: the convergence order is about 0.5 for first-order schemes (respectively $2 / 3$ for second-order schemes) for the variables which are not Riemann invariants of the contact discontinuity (here $\rho$ and $z$ ), while, for the other variables which only vary through shocks (here $\boldsymbol{u}$ and $p$ ), the convergence order is close to 1 (for both first-order and second order schemes), when the scheme exactly preserves the contact discontinuity.
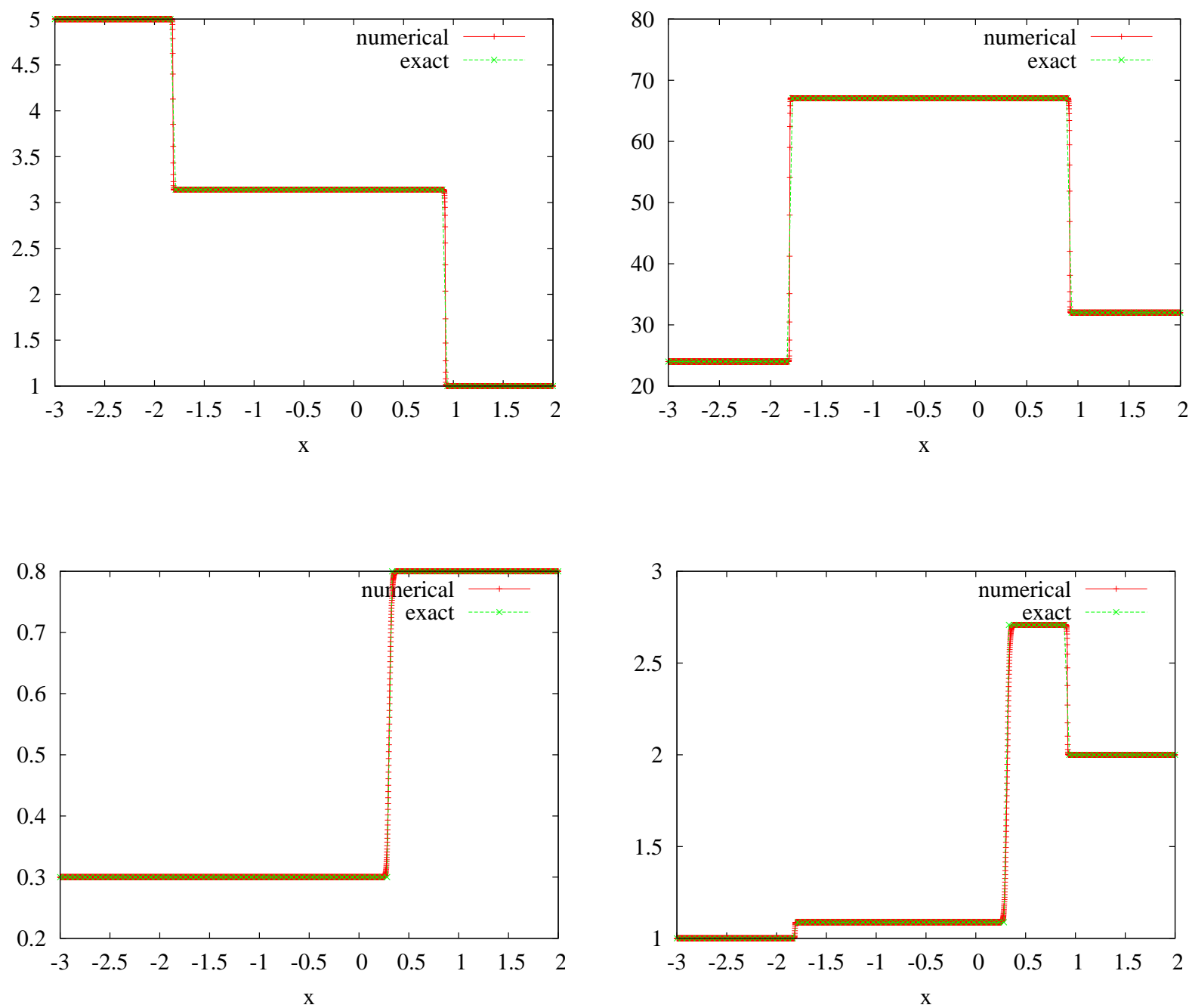

Figure 14: Two-phase test: shock / contact discontinuity / shock - Centred RT scheme Numerical solution at $t=0.1$, with 5000 cells, $\delta t=4.10^{-5}$ (so CFL $=0.75$ ) and $\mu=0.002$. Velocity (top left), pressure (top right), gas mass fraction (bottom left), density (bottom right). 

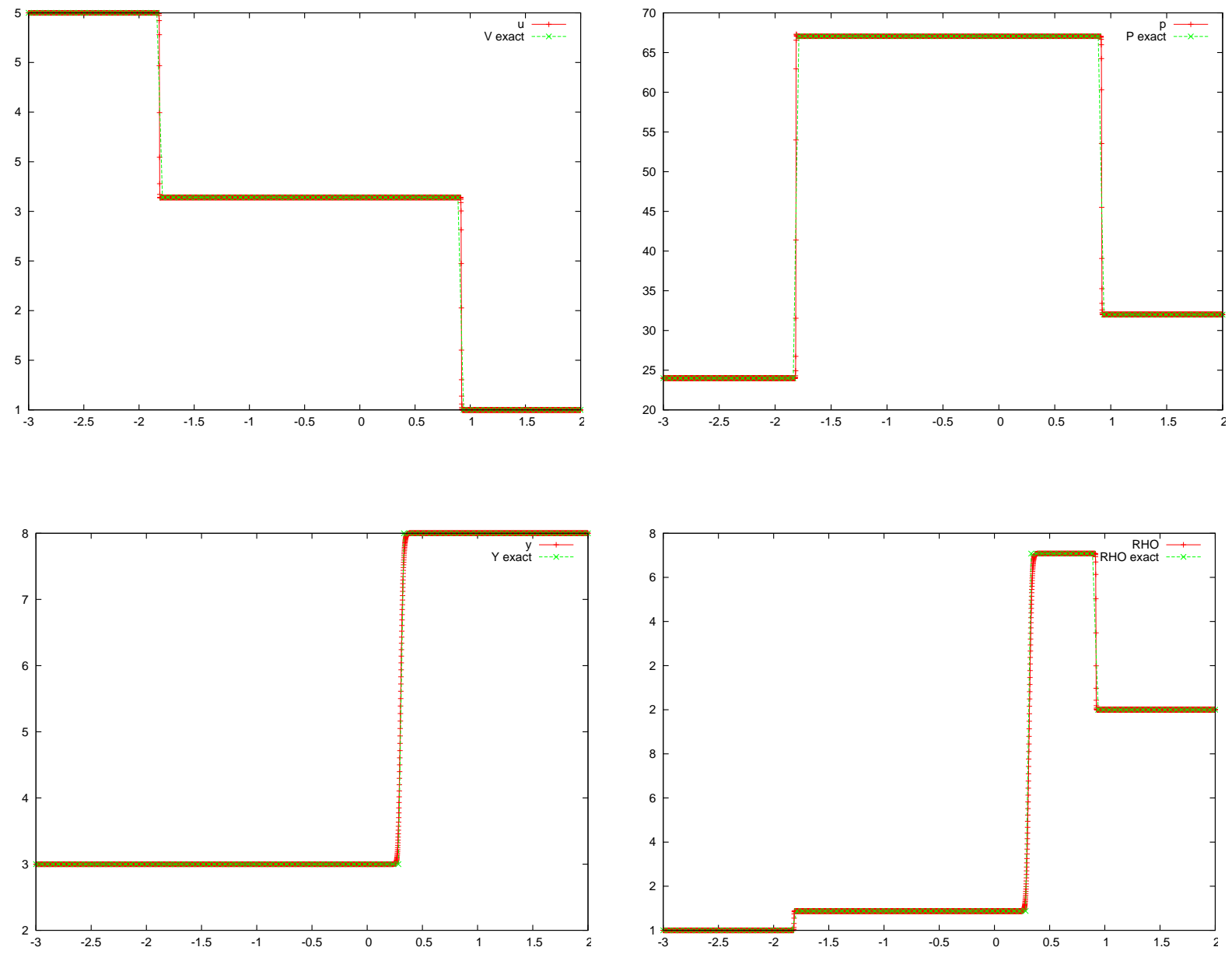

Figure 15: Two-phase test: shock / contact discontinuity / shock - Centred MAC scheme Numerical solution at $t=0.1$, with 5000 cells, $\delta t=4.10^{-5}$ (so $\mathrm{CFL}=0.75$ ) and $\mu=0.002$. Velocity (top left), pressure (top right), gas mass fraction (bottom left), density (bottom right). 



Figure 16: Two-phase test: shock / contact discontinuity / shock - Centred RT scheme Numerical solutions at $t=0.1$ with 2500 cells, $\mu=0.002$, for several CFL numbers. Velocity (top left), pressure (top right), gas mass fraction (bottom left), density (bottom right). 

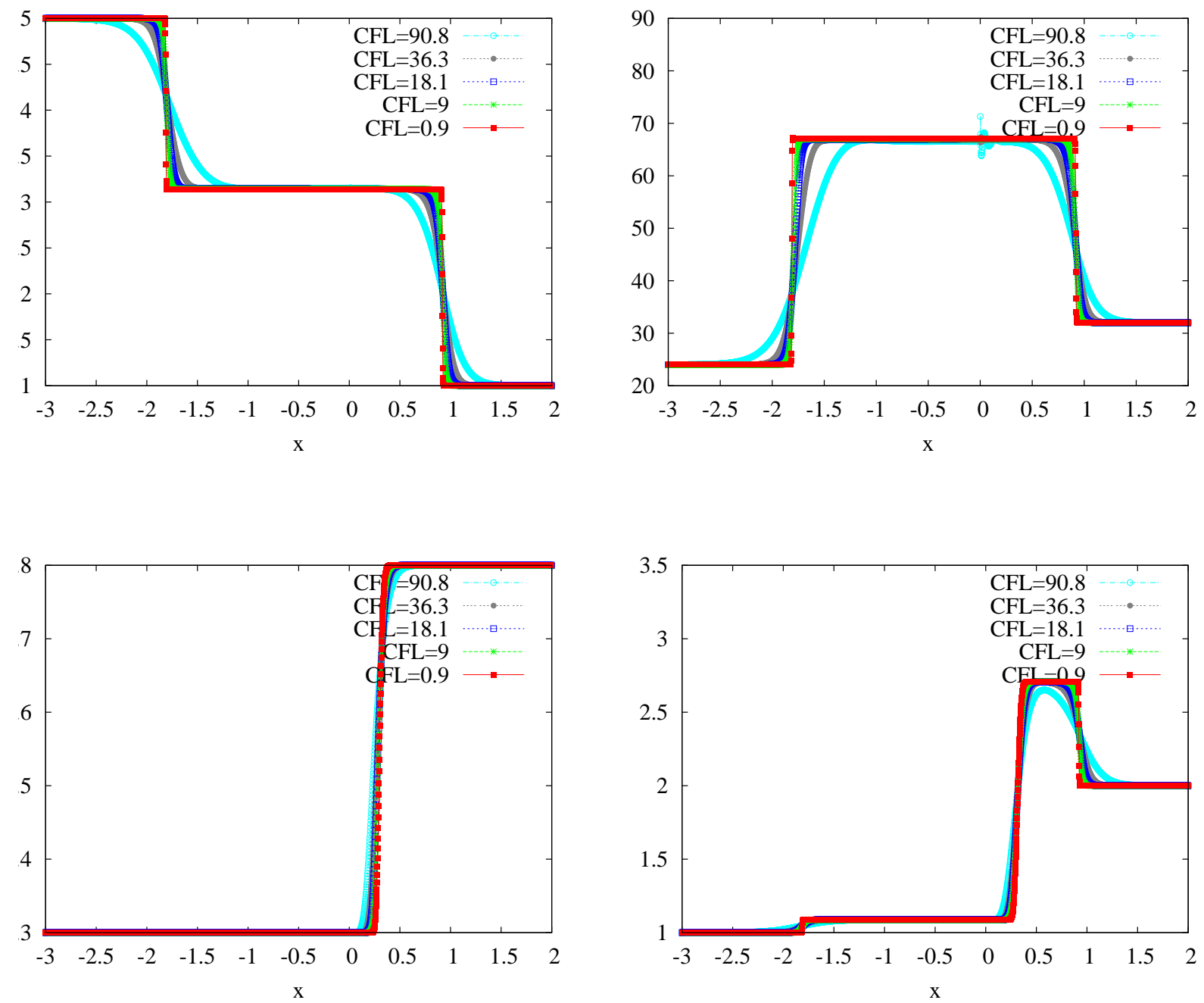

Figure 17: Two-phase test: shock / contact discontinuity / shock - Centred MAC scheme Numerical solutions at $t=0.1$ with 2500 cells, $\mu=0.002$, for several CFL numbers. Velocity (top left), pressure (top right), gas mass fraction (bottom left), density (bottom right). 

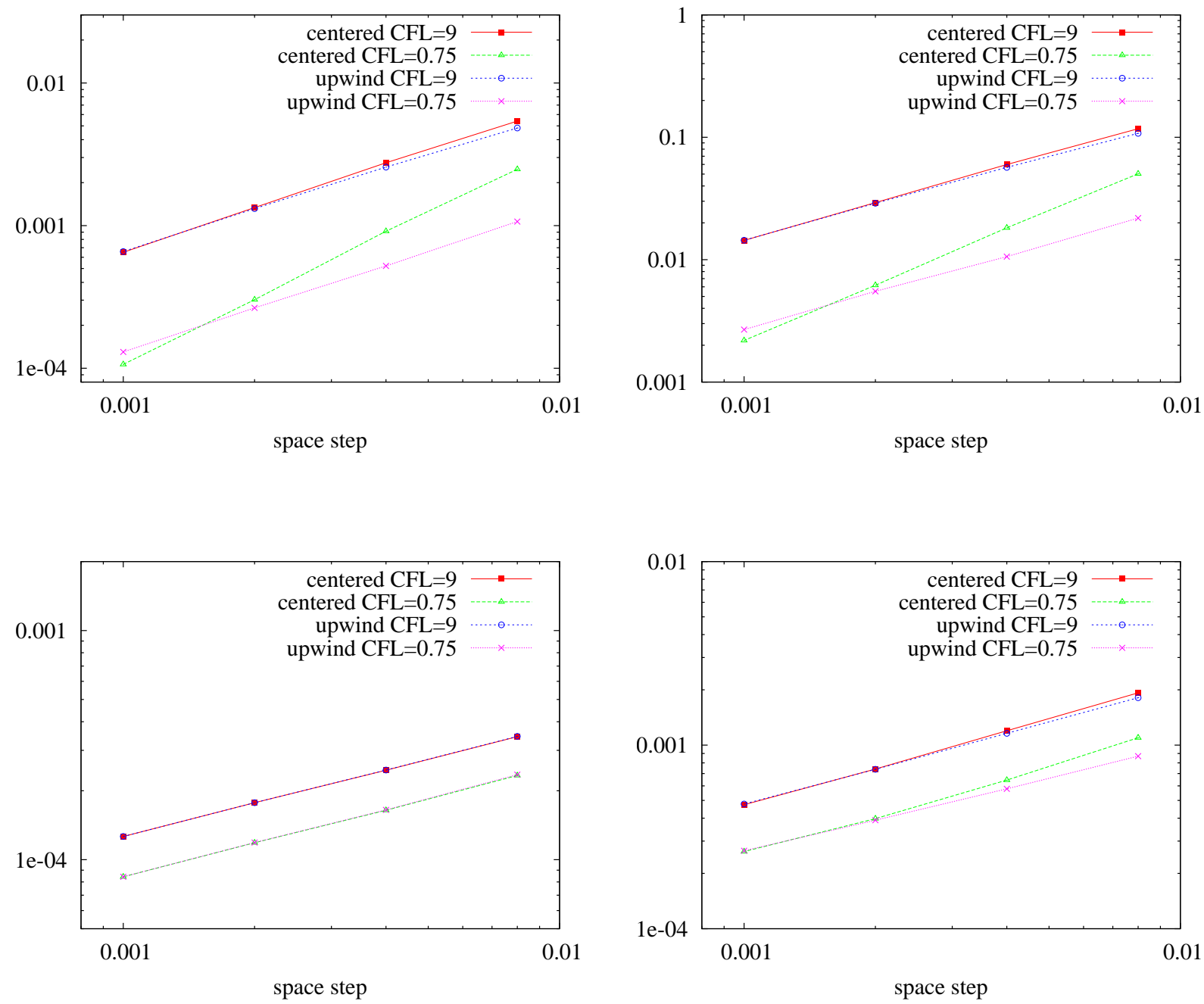

Figure 18: Two-phase test: shock / contact discontinuity / shock - Centred and upwind RT schemes $-L^{1}$ norm of the error at $t=0.1$ between the computed solution and the exact one, as a function of the mesh (or time) step, for two fixed CFL numbers. In the centred case, the used artificial viscosity is $\mu=0.002$, i.e. a value close to the one which yields the most accurate results. Velocity (top left), pressure (top right), gas mass fraction (bottom left) and density (bottom right). 
Pressure correction staggered schemes for barotropic one-phase and two-phase flows
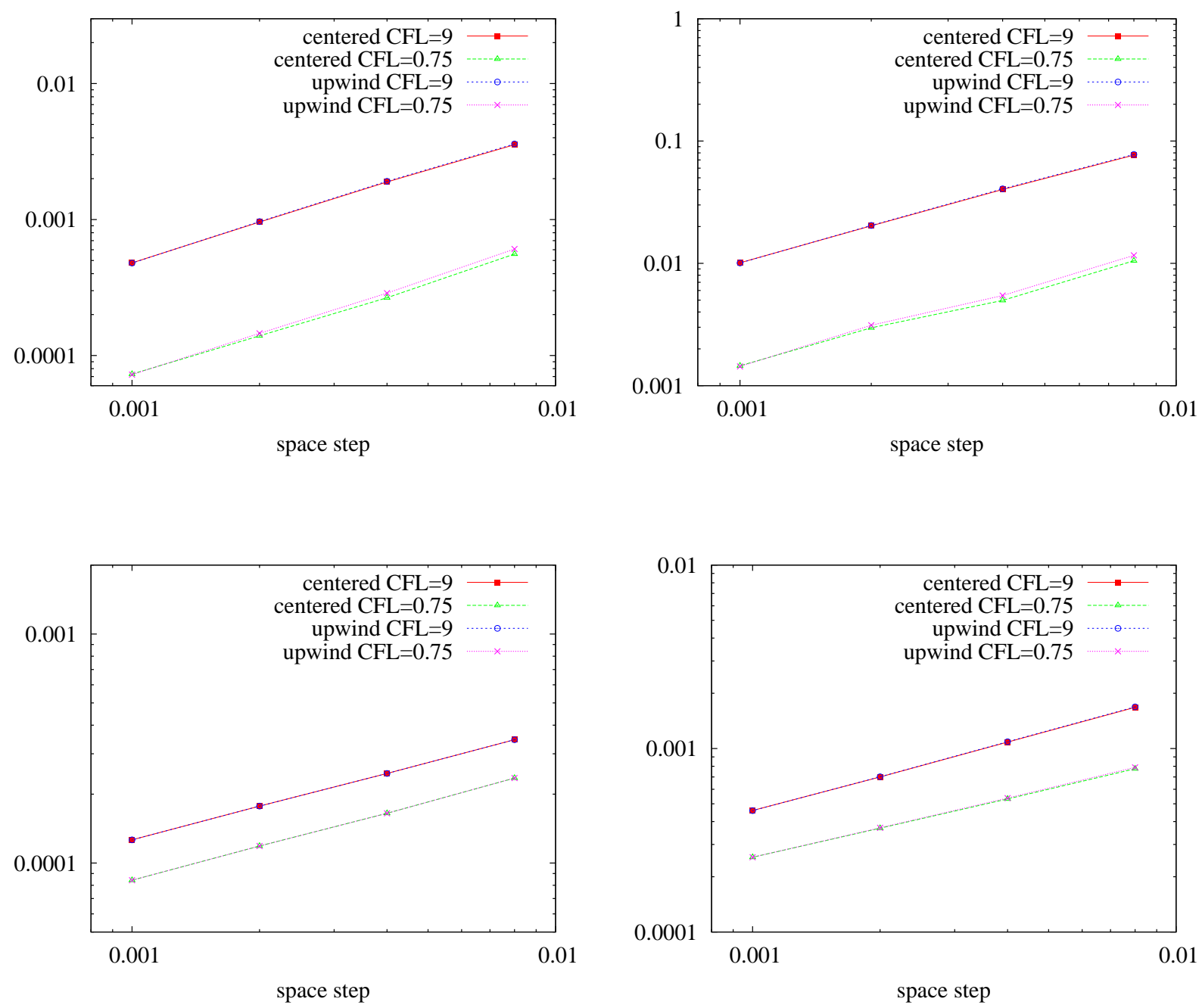

Figure 19: Two-phase test: shock / contact discontinuity / shock - Centred and upwind MAC schemes $-L^{1}$ norm of the error at $t=0.1$ between the computed solution and the exact one, as a function of the mesh (or time) step, for two fixed CFL numbers. In the centred case, the used artificial viscosity is $\mu=0.002$, i.e. the same value as for the RT discretization. Velocity (top left), pressure (top right), gas mass fraction (bottom left) and density (bottom right).

\subsubsection{Second case: rarefaction-contact discontinuity-rarefaction}

We conclude this study by the numerical simulation of a two-phase flow with rarefaction waves. The two initial constant states are given by:

$$
\left(\begin{array}{l}
\rho \\
\boldsymbol{u} \\
y
\end{array}\right)_{L}=\left(\begin{array}{l}
1 \\
0 \\
0.3
\end{array}\right), \quad\left(\begin{array}{l}
\rho \\
\boldsymbol{u} \\
y
\end{array}\right)_{R}=\left(\begin{array}{l}
2 \\
2 \\
0.8
\end{array}\right) .
$$


Pressure correction staggered schemes for barotropic one-phase and two-phase flows

The numerical solutions at $t=0.1$, obtained for 5000 cells, $\delta t=0.0001$ and $\mu=0.002$ with the RT and MAC centred schemes, presented in Figure 20 and Figure 21 respectively, are in close agreement with the exact solution. Convergence studies, performed here only for the centred variants, show similar convergence rates for both the RT and MAC discretizations; for all the variables, the orders of convergence lie in the interval $(0.6,0.8)$, the variables which are discontinuous (resp. continuous) across the contact discontinuity corresponding to the lowest (resp. highest) orders.
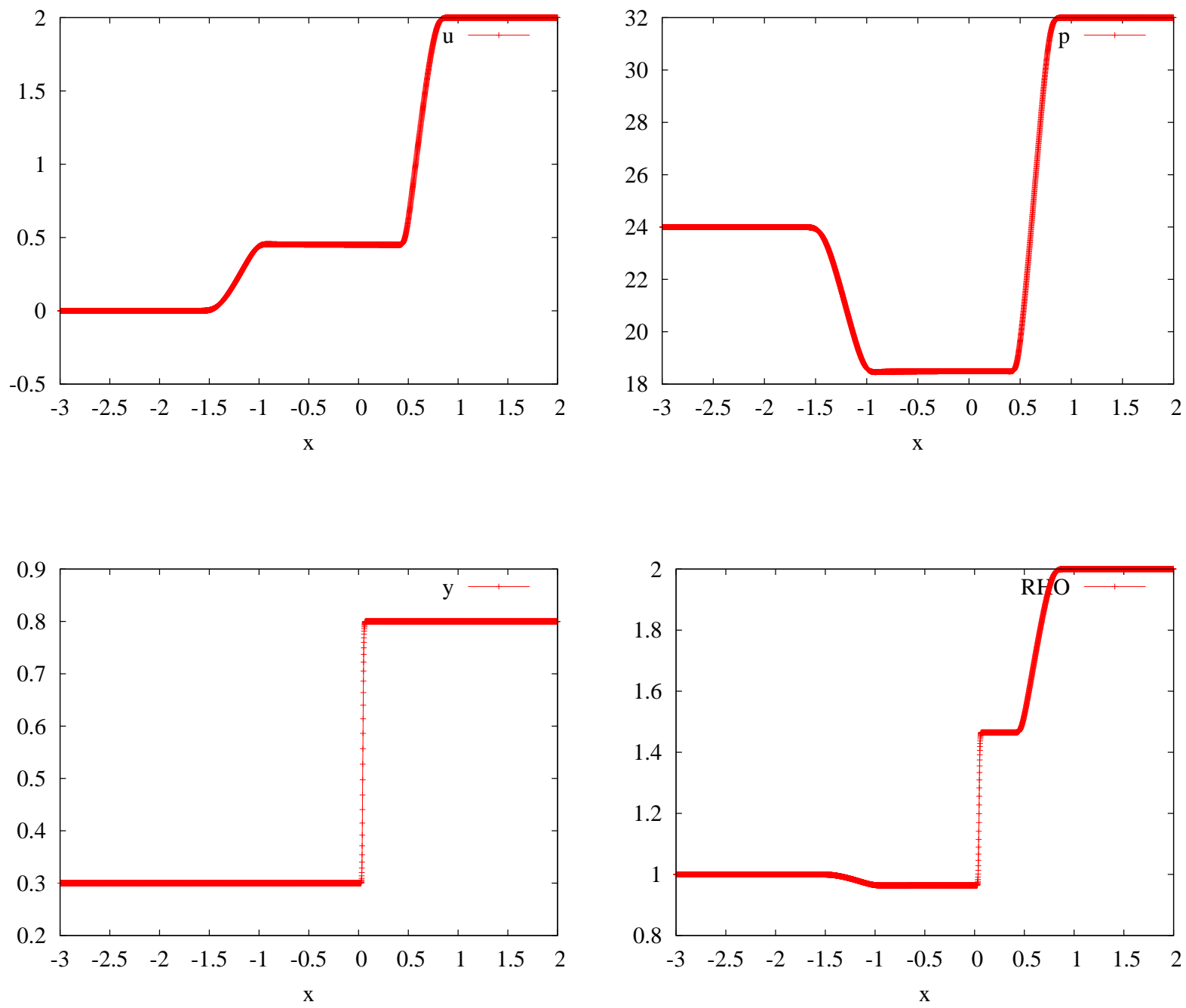

Figure 20: Two-phase test: rarefaction wave / contact discontinuity / rarefaction wave - Centred RT scheme - Numerical solution at $t=0.1$ with 5000 cells, $\delta t=0.0001$ and $\mu=0.002$. Velocity (top left), pressure (top right), gas mass fraction (bottom left), density (bottom right). 

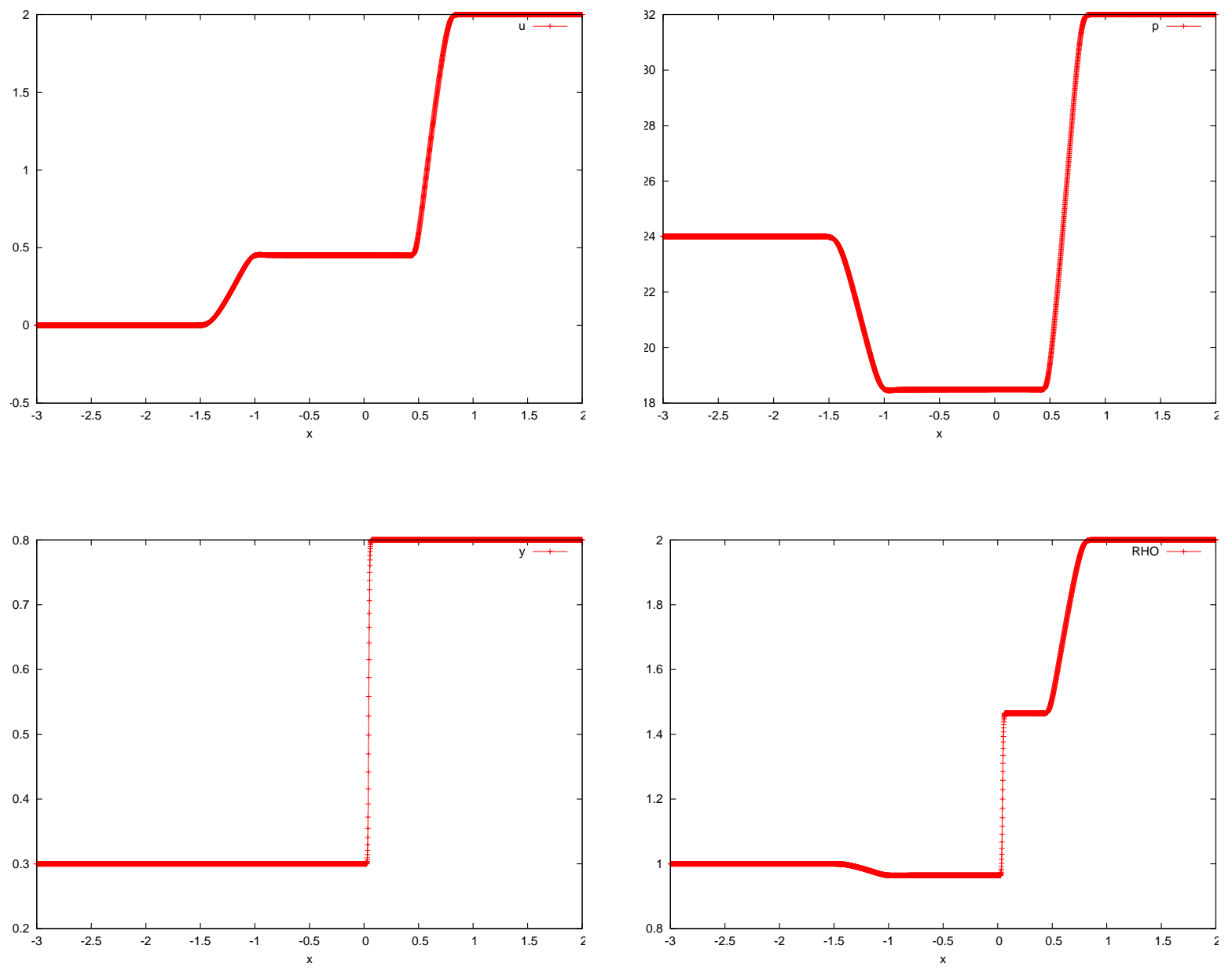

Figure 21: Two-phase test: rarefaction wave / contact discontinuity / rarefaction wave - Centred MAC scheme - Numerical solution at $t=0.1$ with 5000 cells, $\delta t=0.0001$ and $\mu=0.002$. Velocity (top left), pressure (top right), gas mass fraction (bottom left), density (bottom right). 
Pressure correction staggered schemes for barotropic one-phase and two-phase flows

\section{Wind tunnel with a step}

We now turn to two-dimensional test cases. To this purpose, we use in this section a "barotropic one-phase version", which we then extend to obtain a two-phase flow problem, of a test which is classical for (non-barotropic one-phase) Euler equations [29, 13], and is often referred to as "the Mach 3 wind tunnel with step".

The flow enters through the left boundary a L-shaped domain, with a forward facing step, with the following geometry:

$$
\Omega=(0,3) \times(0,1) \backslash(0.6,3) \times(0,0.2) .
$$

All the conservative variables, i.e. $\rho$ and $\rho \boldsymbol{u}$ for the one-phase flow and $\rho, \rho y$ and $\rho \boldsymbol{u}$ in the two-phase case, are prescribed at the inlet (i.e. left) section.

In the one-phase case, the equation of state of the fluid is $p=\rho$ and the inlet values are such that the Mach number is 3, which is obtained here by taking $\boldsymbol{u}=(3,0)^{t}$ and $\rho=1$. On the top and bottom wall, we use symmetry (i.e. perfect slip) boundary conditions. The flow is free at the outflow (right) section, which means, since the resulting Mach number at this boundary is greater than one, that the three eigenvalues of the Jacobian matrix of the system are positive and therefore no boundary condition should be prescribed here; however, since our discretization of the pressure gradient is centred and, less importantly, because we use a physical-like diffusion term in the momentum balance equation, we need an expression for the force which exerts at this surface, which we suppose given by:

$$
\boldsymbol{\tau} \cdot \boldsymbol{n}-p \boldsymbol{n}=p_{0} \boldsymbol{n},
$$

where $p_{0}$ is given the same value than the inlet pressure, i.e. $p_{0}=1$. We discuss later the effects of this boundary condition. The initial condition is $\boldsymbol{u}=(3,0)^{t}$ and $\rho=p=1$.

The mesh is built from a regular $3 n \times n$ Cartesian grid of the rectangle $(0,3) \times(0,1)$, suppressing meshes at the right bottom part of the domain (i.e. $(0.6,3) \times(0 ., 0.2))$ to take the step into account. The computations presented in this section are performed with the centred MAC scheme, and we use an artificial viscosity in the range of what would be the numerical viscosity introduced by an upwinding technique, for the meshes used in this study.

The pressure field obtained with $n=1000$ (i.e. from a $3000 \times 1000$ grid) and $\delta t=10^{-3}$, with a viscosity $\mu=510^{-4}$, is shown in Figure 22. As in the non-barotropic case, we obtain a shock upflow the step, which propagates and reflects on the boundaries. Here, however, the shock moves slowly upward, while it is stationary in the non-barotropic case (so, contrary to this latter case, the flow is not steady at $t=4)$. We studied the mesh convergence and, at $n=1000$, the upper bound of the pressure in the domain (reported in the caption of Figure 22) seems to have reached a value almost independent of the mesh; it is not the case of the lower bound, which is obtained at (or just after) the step corner, and seems to still be mesh and artificial viscosity dependent. Finally, we have to mention that, for the same computation, the upwind scheme with an artificial viscosity equal to zero yields a pressure field showing localized and (probably) spurious structures near the reflection on the bottom of the domain.

Besides the fact that we use, for numerical reasons, a non-physical outflow boundary condition, the time-splitting of pressure correction methods is also known to introduce spurious pressure boundary conditions. It is indeed the case for the present scheme, even if it is derived by an algebraic splitting (i.e. by discretizing first the equations up to obtain an implicit fully discrete scheme and then splitting in time, instead of first writing a split time semi-discrete algorithm 
Pressure correction staggered schemes for barotropic one-phase and two-phase flows

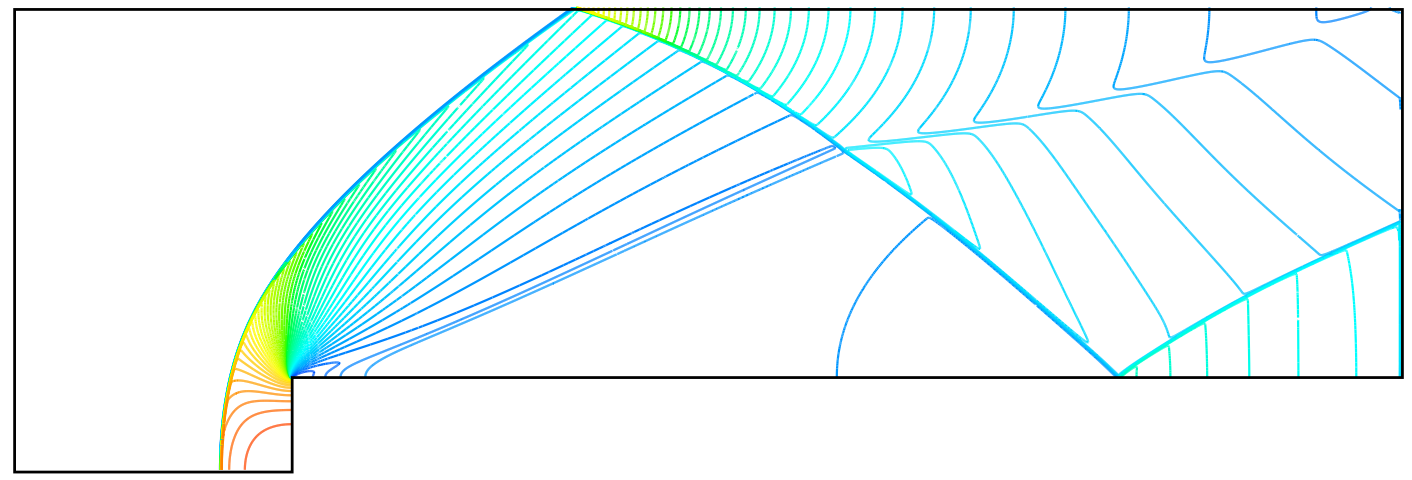

Figure 22: Wind tunnel with step, one-phase case - Centred MAC scheme - Isolines of the pressure field obtained at $\mathrm{t}=4$ with a $3000 \times 1000$ mesh and $\delta t=10^{-3}$. Minimal value (blue): $p=0.37$ - Maximal value (red): $p=9.5$

with (artificial) boundary conditions explicitly stated at each step): we show in [7] that the elliptic problem solved at the correction step for the pressure increment takes the form of a finite volume diffusion problem, with homogeneous Neumann conditions at the boundary where the velocity is prescribed and homogeneous Dirichlet conditions when the velocity is free. In the present case, it means in particular that the pressure suffers from a numerical boundary condition at the outlet section which tends to fix it at the initial value. Note, however, that this boundary condition is only prescribed in the "finite volume way" (i.e. through the expression of the flux), which may be seen as a penalization process with a $\delta t / h$ coefficient, so this condition is relaxed when this latter ratio is small [7]. We observe in Figure 22 that this outlet condition indeed generates a pressure boundary layer. To investigate this phenomenon in more detail, we plot in Figure 23 the value of the pressure along the $x_{2}=3$ line, for various meshes and time steps. We observe that the perturbation of the solution remains localized, and that, as well known for incompressible flow problems, the extension of the affected zone decreases with the space step. Besides, we also see that the computation is at least qualitatively correct for rather coarse meshes and time steps (using $v=|\boldsymbol{u}|+c=4$ with $c$ the speed of sound at the inlet section, $\delta t=0.1$ (resp. $\delta t=0.01)$ corresponds with $n=100$ to $\mathrm{CFL}=40($ resp. $\mathrm{CFL}=4)$ ); in particular, convergence with respect to the time step seems to be reached, for this particular flow, for CFL numbers far greater than 1.

We now turn to a two-phase case, which is obtained by initializing the gas mass fraction by $y=0.1$ for $x_{2} \leq 0.6$ and $y=1$ in the rest of the domain. We choose $\rho_{g}=p$ and $\rho_{\ell}=10$. We recall [14, Relation (93)] that the speed of sound is given, in the two-phase case, by:

$$
c^{2}=\frac{\partial_{p}\left(\varrho_{g}\right) \rho_{\ell}^{2} y}{\left(\rho_{\ell}+z-\rho\right)^{2}},
$$

with $z=\rho y$ and, here, $\partial_{p}\left(\varrho_{g}\right)=1$. This relation shows that the speed of sound is lower for $y=0.1(c \approx 0.6)$ than for $y=1(c=1)$, and we adjust the inlet velocity to keep the value of the Mach number at 3 in the two-phase zone. Inlet conditions are then given by $\boldsymbol{u}=(3 c(y=0.1), 0)^{t}$, $y=0.1$ for $x_{2} \in(0,0.6)$ and $y=1$ for $x_{2} \in(0.6,1)$, and $\rho$ given by the equation of state of the mixture with the local value of $y$ and $p=1$.

As a first step, we only solve the equations with $y(\boldsymbol{x})$ fixed at its initial value and independent of time (doing so, we compute in fact a barotropic flow in a medium with a space-dependent 

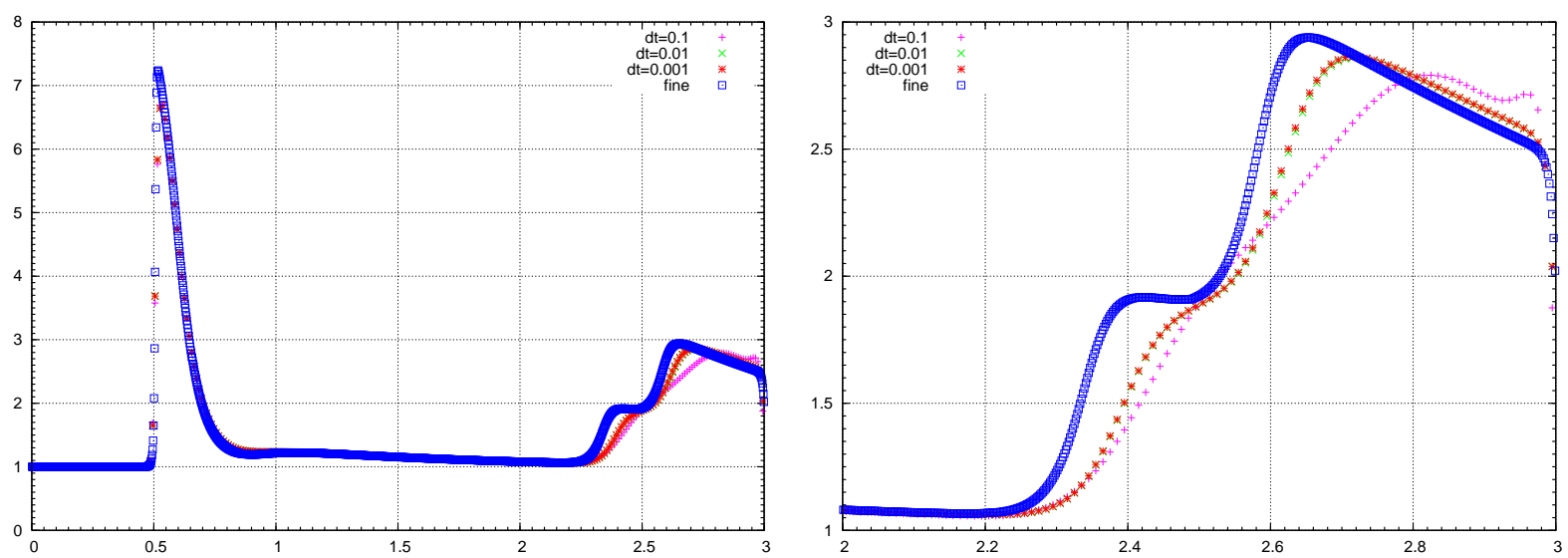

Figure 23: Wind tunnel with step, one-phase case - Centred MAC scheme - Pressure obtained at $\mathrm{t}=4$ along the line $x_{2}=0.3$, with an $300 \times 100$ mesh and various time steps, and with a $1500 \times 500$ mesh and $\delta t=2 \cdot 10^{-3}$.

equation of state). As a consequence of this change of equation of state, we observe that the shock moves upward more rapidly, and interaction with the inlet boundary conditions occurs as soon as $t \approx 2$; consequently, we restrict the time interval of computation, and stop at $t=1.6$. The final time pressure, with a mesh built from the $1500 \times 500$ grid, $\delta t=210^{-3}$ and $\mu=10^{-3}$, is shown in Figure 24. We observe that part of the pressure waves reflects at the $y$ transition, the reflected wave propagating in a direction almost parallel to the transition (i.e. horizontal), thus giving a quite complicated structure. The pressure is very small just after the step corner, and its lowest value still decreases when refining the mesh; once again, the upper bound of the pressure in the domain is much less sensitive to the discretization parameters.

Finally, we perform the same computation with the whole set of equations governing the twophase flow. The obtained pressure, still at $t=1.6$ and with the same grid, time step and artificial viscosity, is shown in Figure 25. The fist part of the flow shows some similarities with the previous computation, but the pressure evolution is quite different downstream, due to the fact that the liquid phase is now transported by the flow. 


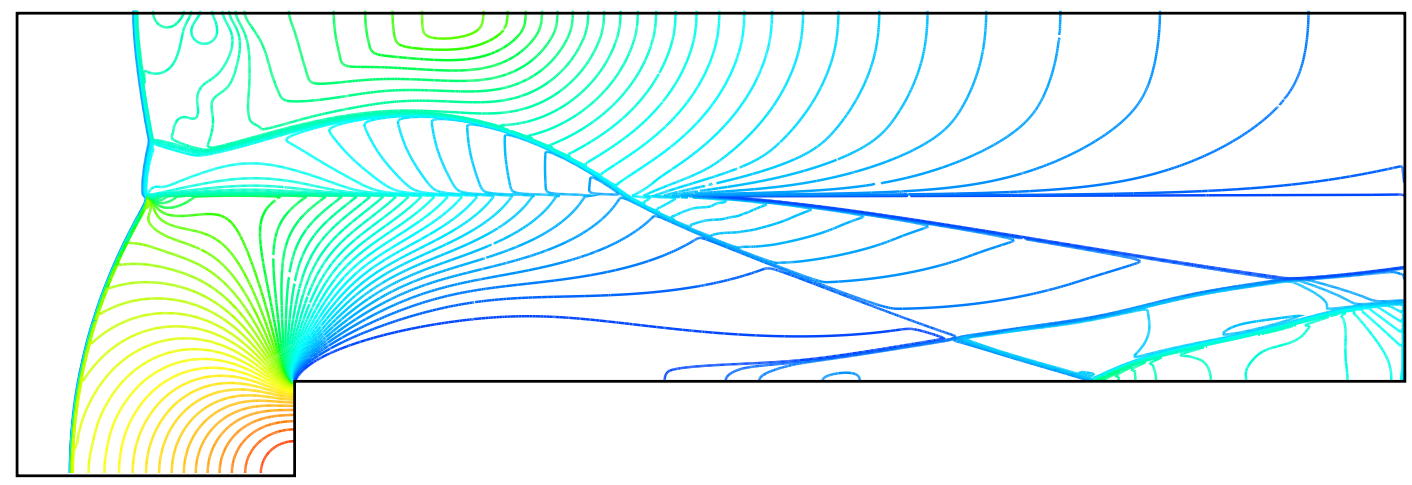

Figure 24: Wind tunnel with step, non-uniform $y$ case - Centred MAC scheme - Isolines of the pressure field obtained at $\mathrm{t}=1.6$ with a $1500 \times 500$ mesh and $\delta t=2 \cdot 10^{-3}$. Minimal value (blue): $p=0.003-$ Maximal value (red): $p=14.0$

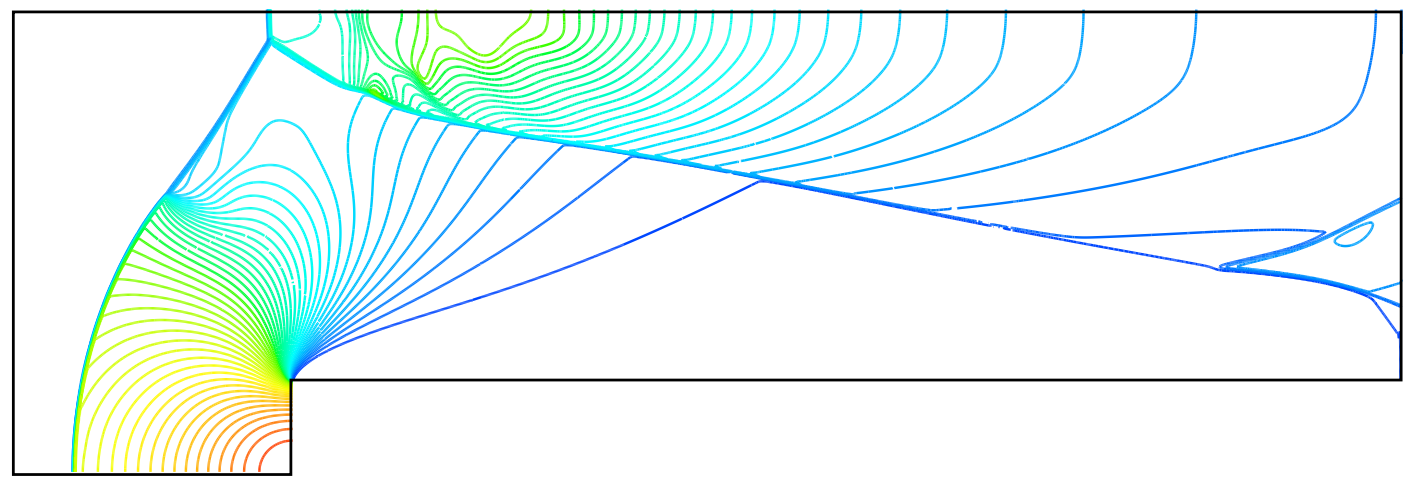

Figure 25: Wind tunnel with step, two-phase case - Centred MAC scheme - Isolines of the pressure field obtained at $\mathrm{t}=1.6$ with a $1500 \times 500$ mesh and $\delta t=2.10^{-3}$. Minimal value (blue): $p=0.003-$ Maximal value (red): $p=13.8$. 
Pressure correction staggered schemes for barotropic one-phase and two-phase flows

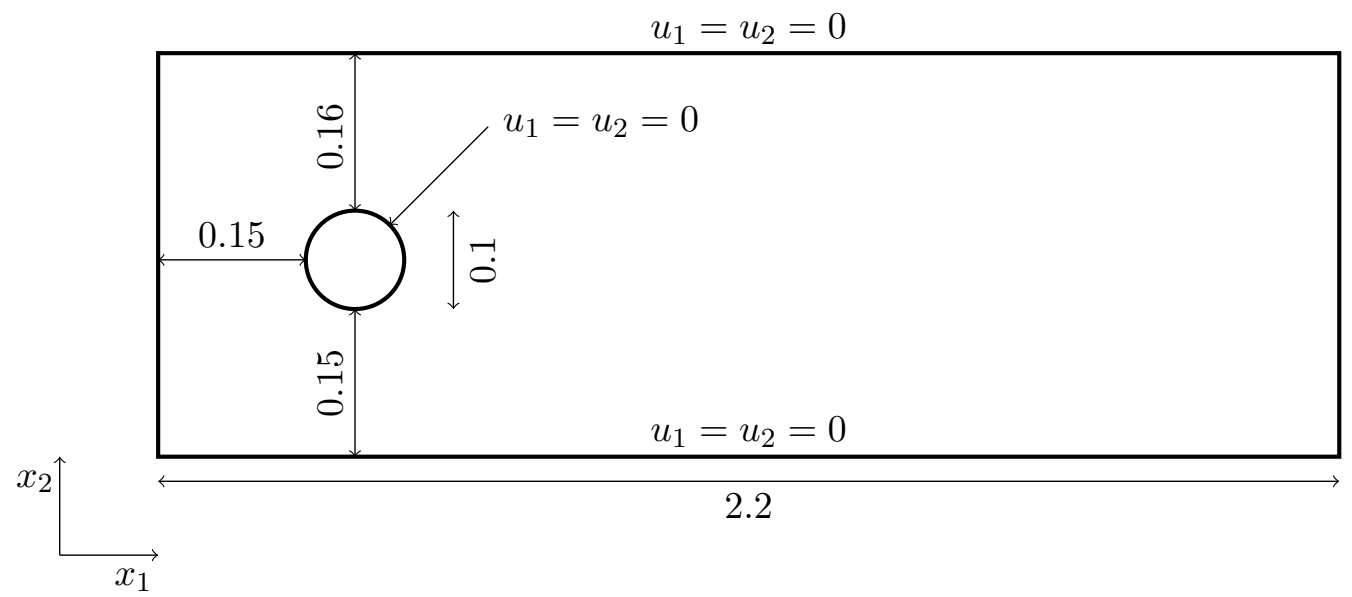

Figure 26: 2D flow around a cylinder - Geometry.

\section{Flow past a cylinder}

We address in this section two test cases which are derived from a benchmark referred to as the 2D-2 case in [26], which consists in the computation of a one-phase two-dimensional flow past a cylinder. The mesh is thus unstructured, and the space discretization is based on the RT element. We suppose that the equation of state reads:

$$
y=1, \rho=\varrho_{g}(p), \text { with } \varrho_{g}=\wp^{-1} \text { and } \wp(s)=\frac{9.81}{2} s^{2} .
$$

so that the problem may be identified to the so-called shallow-water equations, with $\rho$ the water depth.

Our study is split into two parts. In the first step, we address conditions in which the wave celerity is far greater than the material velocity; the flow is thus "almost incompressible", and we can compare our results to the reference ones provided in [26]. In a second step, we investigate a configuration where compressibility effects are dominant.

\subsection{A low Mach number case}

The geometry is sketched on Figure 26. A disk of diameter $D=0.1$ is located inside a 2D channel (the axis of the cylinder lying slightly below the centre line of the channel) and the fluid enters the domain on the left boundary, with an imposed parabolic velocity profile:

$$
u_{1}\left(0, x_{2}\right)=4 u_{m} x_{2} \frac{H-x_{2}}{H^{2}}, \quad u_{2}\left(0, x_{2}\right)=0,
$$

where $H=0.41 \mathrm{~m}$ is the height of the channel and $u_{m}=1.5$. The velocity is prescribed to zero at the other boundaries except the right-hand side, where the flow leaves the domain; in our computation, we impose on this latter boundary a Neumann condition. The fluid density at the initial state and at the inlet boundary is $\rho=1000$, and the constant viscosity is set to $\mu=\rho / 1000=1$. Since the celerity of waves $c \simeq 100\left(c^{2}=9.81 \rho\right)$, the Mach number is close to 0.01 , and the flow is almost incompressible. 
Pressure correction staggered schemes for barotropic one-phase and two-phase flows

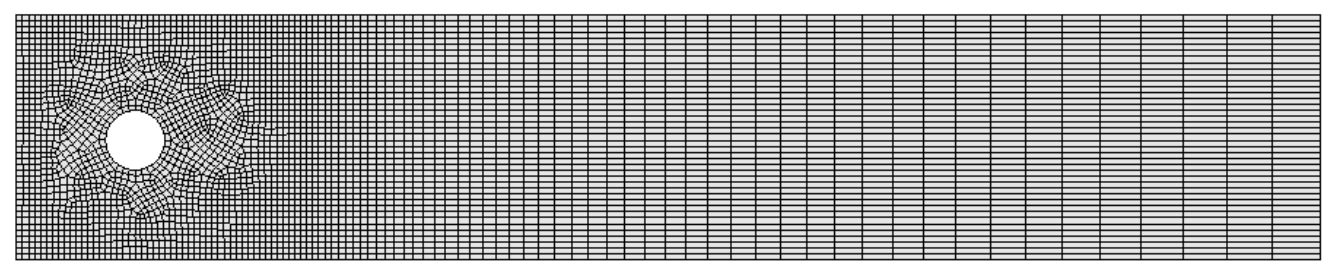

Figure 27: A coarser version of the mesh.

The flow is unsteady, with a periodic shedding of vortices behind the cylinder. The characteristic flow quantities quoted in [26] are the maximum drag coefficient $c_{D \max }$, the maximum lift coefficient $c_{L \max }$ and the Strouhal number St (see [26, section 2.2]). Drag and lift coefficients are defined as follows. First, the drag and lift forces are expressed as:

$$
F_{D}=\int_{S}\left(\rho \nu \frac{\partial u_{t}}{\partial \boldsymbol{n}} n_{2}-p n_{1}\right) d S, \quad F_{L}=-\int_{S}\left(\rho \nu \frac{\partial u_{t}}{\partial \boldsymbol{n}} n_{1}+p n_{2}\right) d S
$$

where $S$ stands for the boundary of the cylinder and $u_{t}$ for the tangential component of the velocity (namely $u_{t}=\boldsymbol{u} \cdot \boldsymbol{t}$ with $\boldsymbol{t}$ the tangential vector to $S$ ). Then, $c_{D}$ and $c_{L}$ are obtained by scaling $F_{D}$ and $F_{L}$ by $2 /\left(\rho \bar{u}^{2} D\right)$. The Strouhal number is $\mathrm{St}=D f / \bar{u}, f$ being the frequency of separation, evaluated from the time evolution of the lift coefficient.

A "coarse version" of the mesh used for the computation is given on Figure 27; the actual mesh is refined with respect to this one, by prescribing the discretization step to smaller values along some characteristic lines (the boundaries and concentric circles around the cylinder). The numbers of cells is close to $10^{5}$, and the computation is performed with $\delta t=10^{-4}$.

From a qualitative point of view, we obtain the expected vortex shedding phenomenon. The density is almost constant in the flow, with deviations from the inlet value $\rho=1000$ which lie in the interval $(-0.13,0.21)$. The computed maximum drag and lift coefficients and the Strouhal number are $c_{D}=3.24, c_{L}=1.01$ and $\mathrm{St}=0.300$; these values belong to the reference intervals determined from the benchmark exercise [26], namely $(3.22,3.24),(0.99,1.01)$ and $(0.295,0.305)$ respectively. They are also close to the values obtained with an algorithm based on the same space discretization but solving the equations of the asymptotic model for low Mach number flows [4].

\subsection{A compressible case}

We now adapt the data to obtain a flow governed by compressibility effects, by just lowering the density (or water depth) range to the order of unity (and so the wave celerity also, to the same range). The computational domain is enlarged by adding the surface $(-0.8,0) \times(0, H)$ and, at the initial time, the density and velocity are given by:

$$
\text { for } x_{1} \leq 0, \rho=1 \text { and } \boldsymbol{u}=\left[\begin{array}{c}
u_{L} \\
0
\end{array}\right] ; \text { for } x_{1}>0, \rho=0.5 \text { and } \boldsymbol{u}=\left[\begin{array}{l}
0 \\
0
\end{array}\right]
$$

with $u_{L}=1.9180067779$. The boundary conditions at the top and bottom lines are perfect slip conditions. At the inlet (left) boundary, $\rho$ is prescribed to 1 and the velocity is prescribed to 
Pressure correction staggered schemes for barotropic one-phase and two-phase flows
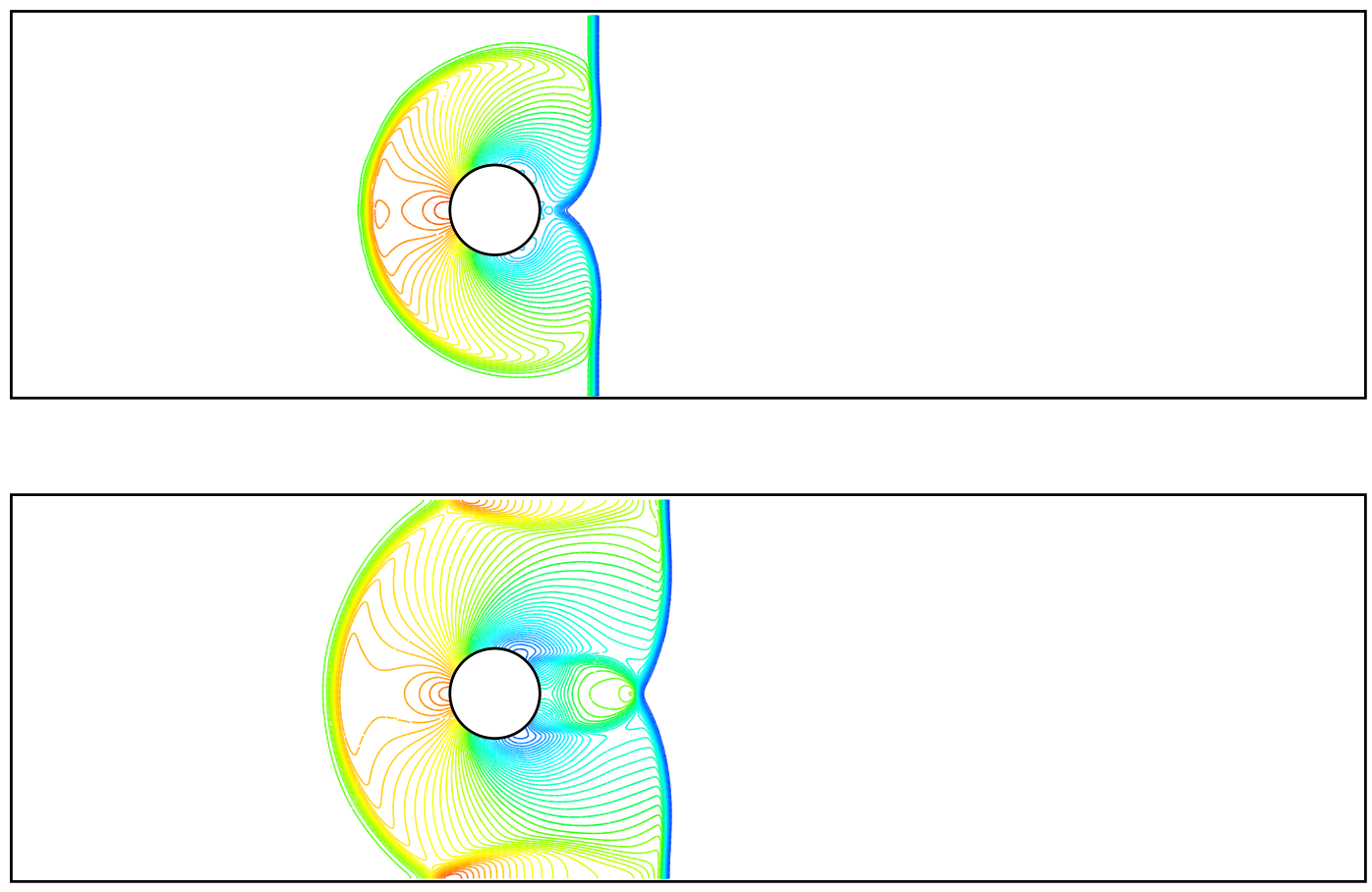

Figure 28: Flow past a cylinder $(1 / 3)$ - Isolines of the pressure field obtained at $t=0.08$ (top) and $t=0.1$ (bottom). The minimal value (blue) of the pressure is $p=1.23$ (value at the right state) - The maximal values (red) are $p=8.57$ (top) and $p=8.24$ (bottom).

$\left(u_{L}, 0\right)^{t}$. Without the disk and for an inviscid fluid, the solution of the problem thus would be a $1 \mathrm{D}$ flow with only one (non-zero amplitude) shock wave traveling to the left at the velocity $2 u_{L}$ and connecting the left and right initial states. The considered final time is $T=0.28$. At this time, the leading shock does not reach the right boundary, so the velocity remains to zero here all along the computation, so we can use a homogeneous Dirichlet boundary condition without perturbing the flow. Finally, we keep the same density to viscosity ratio as in the previous study, so $\mu=0.001$, and no-slip conditions are prescribed on the boundary of the cylinder.

The mesh used for this study is composed of about $410^{5}$ cells. For $x_{1} \geq 0$, it is built from a coarse mesh as sketched on Figure 27, by splitting each cells in sub-quadrangles; for $x_{1} \leq 0$, we use a uniform grid, which has the same edges as the "right mesh" along the line $\{0\} \times(0, H)$, so that the resulting composite mesh is conforming. The time step is $\delta t=10^{-4}$.

The pressure obtained at various times is sketched on Figures 28-30. We observe an interaction of the shock with the cylinder and the walls which generates multiple reflections. At the end of the transient, it seems that shocks are rather smeared, and an extension of the scheme to more accurate (MUSCL-like, for instance) discretizations should be suitable; this work is to be undertaken in a next future. 

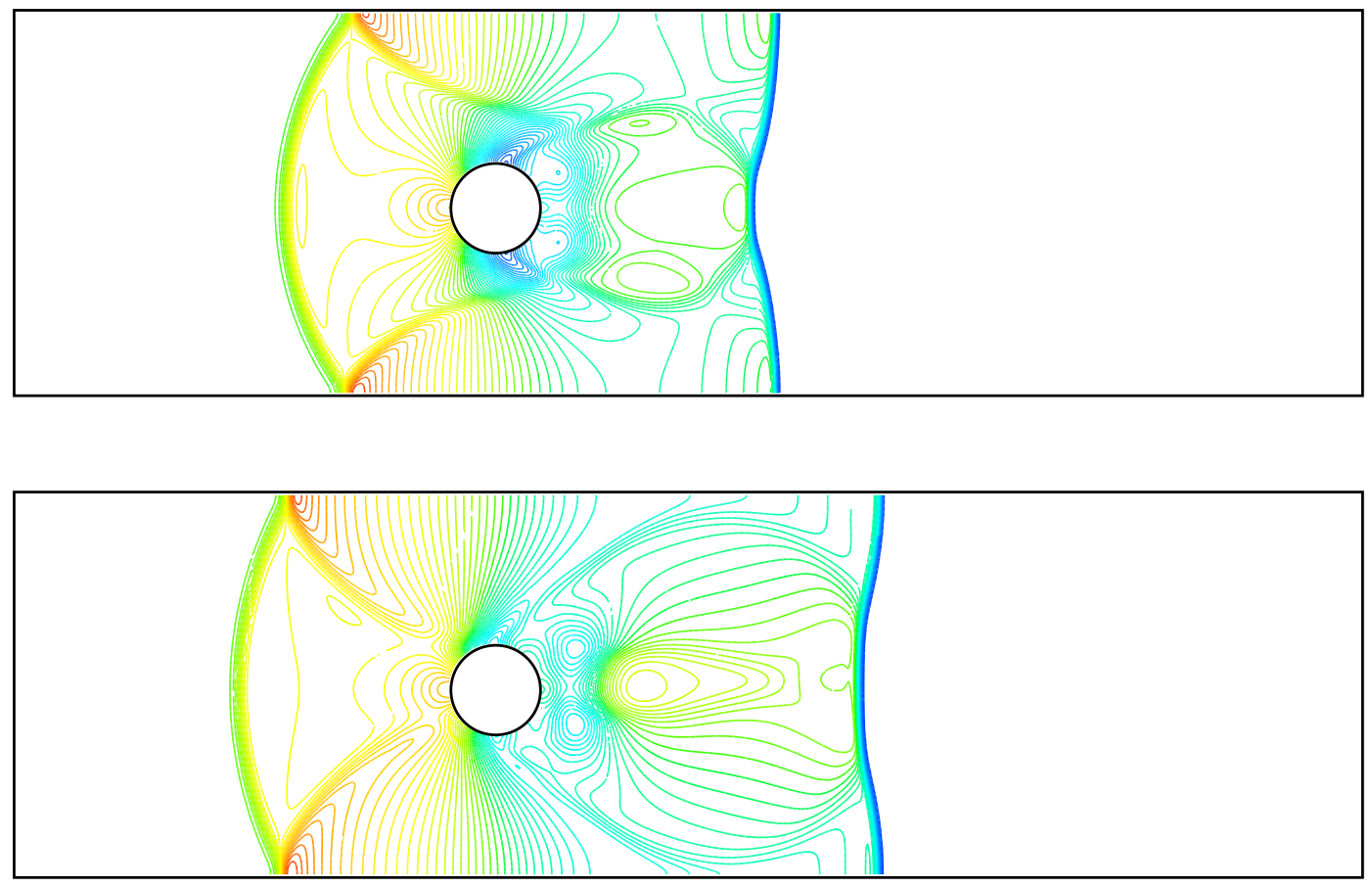

Figure 29: Flow past a cylinder $(2 / 3)$ - Isolines of the pressure field obtained at $t=0.13$ (top) and $t=0.16$ (bottom). The minimal value (blue) of the pressure is $p=1.23$ (value at the right state) - The maximal values (red) are $p=8.56$ (top) and $p=8.35$ (bottom). 

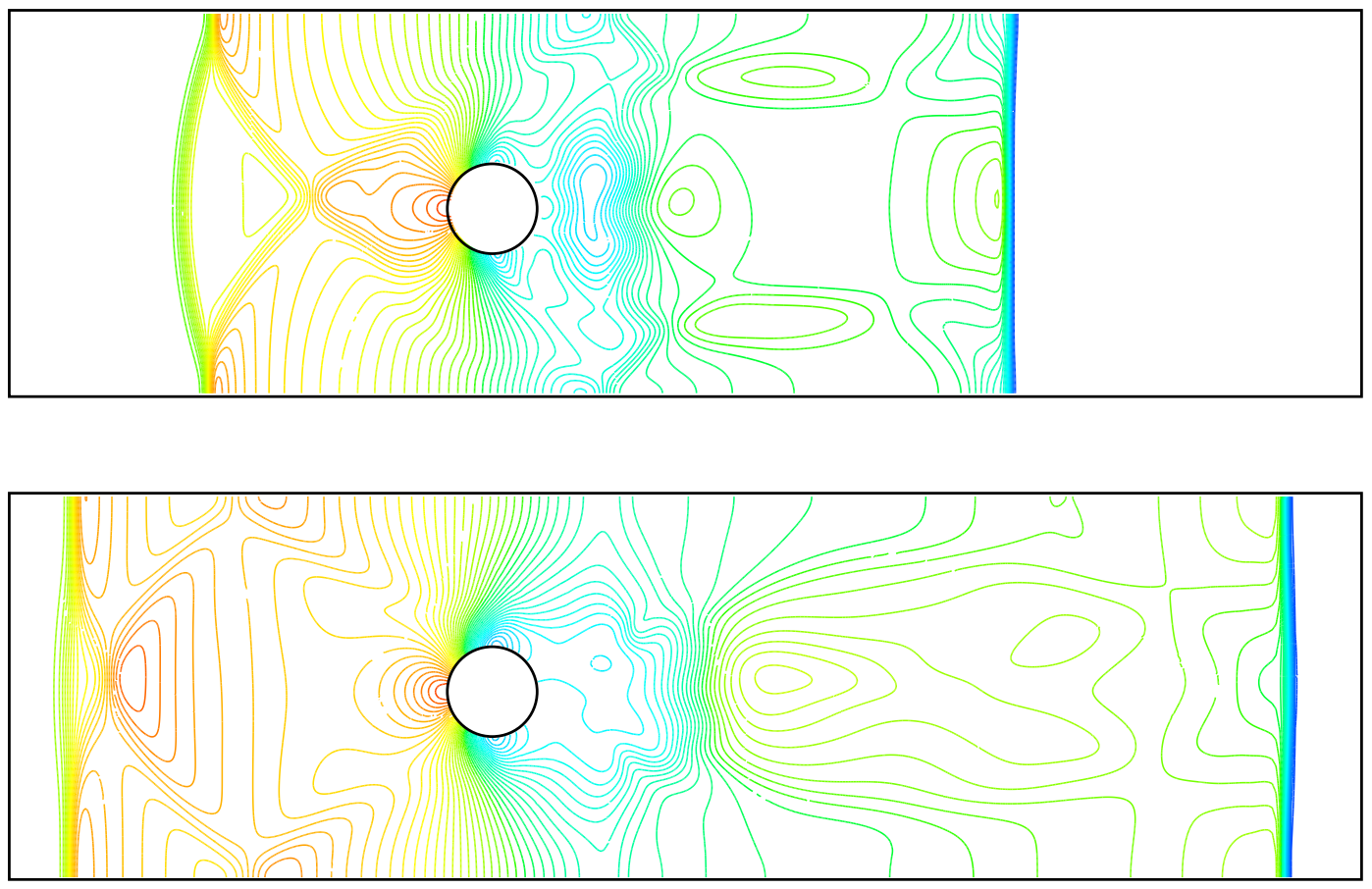

Figure 30: Flow past a cylinder $(3 / 3)$ - Isolines of the pressure field obtained at $t=0.2$ (top) and $t=0.28$ (bottom). The minimal value (blue) of the pressure is $p=1.23$ (value at the right state) - The maximal values (red) are $p=8.36$ (top) and $p=7.58$ (bottom). 
Pressure correction staggered schemes for barotropic one-phase and two-phase flows

\section{Conclusion}

In this paper, we have assessed the capability of a scheme issued from the incompressible flow context, namely a pressure correction scheme, to compute discontinuous solutions of hyperbolic systems. This scheme was already known to be unconditionally stable $[8,10]$. Numerical tests performed here show that, provided that a sufficient numerical dissipation is introduced in the scheme, it converges to the (weak and entropic) solution to the continuous problem; in addition, it shows a satisfactory behaviour up to large CFL numbers. Since the scheme boils down to a usual projection scheme when the density is constant, this approach yields an algorithm which is robust with respect to the flow Mach number, and the present solver is indeed now routinely used to compute viscous two-phase low Mach number flows, as bubble columns for instance.

The present work may be extended in various ways. First, the observed convergence can be comforted by theoretical arguments; even if a complete convergence proof seems difficult at this time, because of the lack of compactness of sequences of discrete solutions due, in particular, to the absence of diffusion terms, it is possible to show, for one-phase flows, by passing to the limit in the scheme, that any limit of a convergent sequence is an entropy weak solution to the continuous problem [17].

Second, several variants of the scheme may be envisaged. The time discretization may be changed to an explicit one, to compute highly transient flows where a time-step limitation is not too stringent in practice; such a scheme has been implemented, and first numerical result are promising. The extension of the above mentioned theoretical results to such a time discretization, under stability restrictions, seems possible. Third, in its present state, the scheme appears to be rather diffusive. Several directions exist to cure this problem. For instance, the artificial viscosity necessary for the scheme to converge could be monitored by a posteriori indicators, following the ideas developed in $[12,13]$. Another route, especially for the explicit variant of the scheme, is to implement MUSCL techniques; this work is underway.

Finally, let us mention that the present scheme has been extended to usual (i.e. non-barotropic) Euler and Navier-Stokes equations, with a quite similar numerical behaviour and theoretical basis [17].

\section{References}

[1] G. Ansanay-Alex, F. Babik, J.-C. Latché, and D. Vola. An L2-Stable Approximation of the Navier-Stokes Convection Operator for Low-Order Non-Conforming Finite Elements. International Journal For Numerical Methods In Fluids, 66:555-580, 2011.

[2] F. Archambeau, J.-M. Hérard, and J. Laviéville. Comparative study of pressurecorrection and Godunov-type schemes on unsteady compressible cases. Computers $\& 3$ Fluids, 38(8):1495-1509, 2009.

[3] F. Babik, R. Herbin, W. Kheriji, and J.-C. Latché. Discretization of the viscous dissipation term with the MAC scheme. In Finite Volumes for Complex Applications VI - Problems and Perspectives - Prague, volume 1, pages 571-579, 2011.

[4] F. Boyer, F. Dardalhon, C. Lapuerta, and J.-C. Latché. Stability of a Crank-Nicolson pressure correction scheme based on staggered discretizations. to appear in International Journal for Numerical Methods in Fluids, 2013. 
Pressure correction staggered schemes for barotropic one-phase and two-phase flows

[5] P. G. Ciarlet. Handbook of numerical analysis volume II : Finite elements methods Basic error estimates for elliptic problems. In P. Ciarlet and J. Lions, editors, Handbook of Numerical Analysis, Volume II, pages 17-351. North Holland, 1991.

[6] M. Crouzeix and P. Raviart. Conforming and nonconforming finite element methods for solving the stationary Stokes equations. RAIRO Série Rouge, 7:33-75, 1973.

[7] F. Dardalhon, J.-C. Latché, and S. Minjeaud. Analysis of a projection method for low-order non-conforming finite elements. IMA Journal of Numerical Analysis, 33:295-317, 2013.

[8] T. Gallouët, L. Gastaldo, R. Herbin, and J.-C. Latché. An unconditionally stable pressure correction scheme for compressible barotropic Navier-Stokes equations. Mathematical Modelling and Numerical Analysis, 42:303-331, 2008.

[9] L. Gastaldo, R. Herbin, W. Kheriji, C. Lapuerta, and J.-C. Latché. Staggered discretizations, pressure correction schemes and all speed barotropic flows. In Finite Volumes for Complex Applications VI - Problems and Perspectives - Prague, Czech Republic, volume 2, pages 39-56, 2011.

[10] L. Gastaldo, R. Herbin, and J.-C. Latché. An unconditionally stable finite element-finite volume pressure correction scheme for the drift-flux model. Mathematical Modelling and Numerical Analysis, 44:251-287, 2010.

[11] E. Godlewski and P. Raviart. Numerical analysis of hyperbolic systems of conservation laws, 1996.

[12] J. Guermond and R. Pasquetti. Entropy-based nonlinear viscosity for Fourier approximations of conservation laws. Comptes Rendus de l'Académie des Sciences de Paris - Série I - Analyse Numérique, 346:801-806, 2008.

[13] J. Guermond, R. Pasquetti, and B. Popov. Entropy viscosity method for nonlinear conservation laws. Journal of Computational Physics, 230:4248-4267, 2011.

[14] H. Guillard and F. Duval. A Darcy law for the drift velocity in a two-phase flow model. Journal of Computational Physics, 224:288-313, 2007.

[15] F. Harlow and A. Amsden. A numerical fluid dynamics calculation method for all flow speeds. Journal of Computational Physics, 8:197-213, 1971.

[16] F. Harlow and J. Welsh. Numerical calculation of time-dependent viscous incompressible flow of fluid with free surface. Physics of Fluids, 8:2182-2189, 1965.

[17] R. Herbin, W. Kheriji, and J.-C. Latché. Consistent semi-implicit staggered schemes for compressible flows. submitted, 2013.

[18] R. Herbin and J.-C. Latché. Kinetic energy control in the MAC discretization of the compressible Navier-Stokes equations. International Journal of Finite Volumes, http://www.latp.univ-mrs.fr/IJFV/, 7, 2010.

[19] ISIS. A CFD computer code for the simulation of reactive turbulent flows. https://gforge.irsn.fr/gf/project/isis. 
Pressure correction staggered schemes for barotropic one-phase and two-phase flows

[20] D. Kuzmin and S. Turek. Numerical simulation of turbulent bubbly flows. In 3rd International Symposium on Two-Phase Flow Modelling and Experimentation, Pisa, 22-24 September, 2004.

[21] B. Larrouturou. How to preserve the mass fractions positivity when computing compressible multi-component flows. Journal of Computational Physics, 95:59-84, 1991.

[22] F. Moukalled, M. Darwish, and B. Sekar. A pressure-based algorithm for multi-phase flow at all speeds. Journal of Computational Physics, 190:550-571, 2003.

[23] R. Panescu. Modélisation Eulérienne d'écoulements diphasiques à phase dispersée et simulation numérique par une méthode volumes - éléments finis. $\mathrm{PhD}$ thesis, Université de Nice Sophia Antipolis, 2006.

[24] PELICANS. Collaborative development environment. https://gforge.irsn.fr/gf/project/pelicans.

[25] R. Rannacher and S. Turek. Simple nonconforming quadrilateral Stokes element. Numerical Methods for Partial Differential Equations, 8:97-111, 1992.

[26] M. Schäfer and S. Turek. Benchmark Computations of Laminar Flow Around a Cylinder. In E. Hirschel, editor, Flow Simulation with High-Performance Computers II, volume 52 of Notes on Numerical Fluid Mechanics, pages 547-566, 1996.

[27] B. Spalding. Numerical computation of multiphase flow and heat transfer. In Recent Advances in Numerical Methods in Fluids - Volume 1, pages 139-168, 1980.

[28] P. Wesseling. Principles of computational fluid dynamics. volume 29 of Springer Series in Computational Mathematics. Springer, 2001.

[29] P. Woodward and P. Colella. The numerical simulation of two-dimensional fluid flow with strong shocks. Journal of Computational Physics, 54:115-173, 1984. 